\title{
An arithmetic Hilbert-Samuel theorem for pointed stable curves
}

Received June 5, 2009 and in revised form January 13, 2010

\begin{abstract}
Let $\left(\mathcal{O}, \Sigma, F_{\infty}\right)$ be an arithmetic ring of Krull dimension at most $1, \mathcal{S}=\operatorname{Spec} \mathcal{O}$ and $\left(\mathcal{X} \rightarrow \mathcal{S} ; \sigma_{1}, \ldots, \sigma_{n}\right)$ a pointed stable curve. Write $\mathcal{U}=\mathcal{X} \backslash \bigcup_{j} \sigma_{j}(\mathcal{S})$. For every integer $k \geq 0$, the invertible sheaf $\omega_{\mathcal{X} / \mathcal{S}}^{k+1}\left(k \sigma_{1}+\cdots+k \sigma_{n}\right)$ inherits a singular hermitian structure from the hyperbolic metric on the Riemann surface $\mathcal{U}_{\infty}$. In this article we define a Quillen type metric $\|\cdot\|_{Q}$ on the determinant line $\lambda_{k+1}=\lambda\left(\omega_{\mathcal{X} / \mathcal{S}}^{k+1}\left(k \sigma_{1}+\cdots+k \sigma_{n}\right)\right)$ and compute the arithmetic degree of $\left(\lambda_{k+1},\|\cdot\|_{Q}\right)$ by means of an analogue of the Riemann-Roch theorem in Arakelov geometry. As a byproduct, we obtain an arithmetic Hilbert-Samuel formula: the arithmetic degree of $\left(\lambda_{k+1},\|\cdot\|_{L^{2}}\right)$ admits an asymptotic expansion in $k$, whose leading coefficient is given by the arithmetic self-intersection of $\left(\omega \mathcal{X} / \mathcal{S}\left(\sigma_{1}+\cdots+\sigma_{n}\right),\|\cdot\|_{\text {hyp }}\right)$. Here $\|\cdot\|_{L^{2}}$ and $\|\cdot\|_{\text {hyp }}$ denote the $L^{2}$ metric and the dual of the hyperbolic metric, respectively. Examples of application are given for pointed stable curves of genus 0 .
\end{abstract}

\section{Contents}

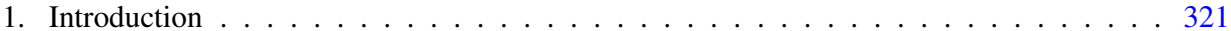

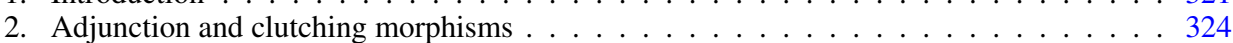

3. Tautological line bundles and Mumford isomorphisms . . . . . . . . . . . . . . . . . . 329

4. Hermitian tautological line bundles . . . . . . . . . . . . . . . . . . . . . . . . . 332

5. Degeneracy of the Quillen metric . . . . . . . . . . . . . . . . . . . . 335

6. Metrized Mumford isomorphisms on $\mathcal{M}_{g, n} \ldots \ldots \ldots$. . . . . . . . . . . . 341

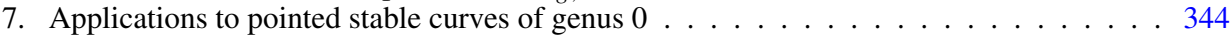

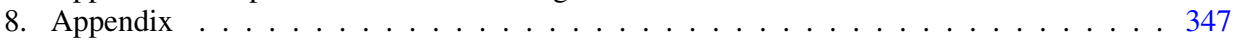

References . . . . . . . . . . . . . . . . . . . . . . . . . 349

\section{Introduction}

Let $\left(\mathcal{O}, \Sigma, F_{\infty}\right)$ be an arithmetic ring of Krull dimension at most 1 [13, Def. 3.1.1]. Set $\mathcal{S}=\operatorname{Spec} \mathcal{O}$ and let $\eta$ stand for its generic point. Let $\left(\pi: \mathcal{X} \rightarrow \mathcal{S} ; \sigma_{1}, \ldots, \sigma_{n}\right)$ be an $n$-pointed stable curve of genus $g$, in the sense of Knudsen and Mumford [22, Def. 1.1]. Assume that $\mathcal{X}_{\eta}$ is smooth. Write $\mathcal{U}=\mathcal{X} \backslash \bigcup_{j} \sigma_{j}(\mathcal{S})$. The connected components of the complex analytic space $\mathcal{U}_{\infty}:=\bigsqcup_{\sigma \in \Sigma} \mathcal{U}_{\sigma}(\mathbb{C})$ are hyperbolic Riemann surfaces of

G. Freixas i Montplet: C.N.R.S., Institut de Mathématiques de Jussieu, 175, Rue du Chevaleret, 75013 Paris Cedex, France; e-mail: freixas@math.jussieu.fr 
finite type. The stability hypothesis guarantees the existence of a unique complete hyperbolic metric of constant curvature -1 on $\mathcal{U}_{\infty}$. This metric induces an arakelovian-i.e. invariant under the action of $F_{\infty}$-hermitian structure $\|\cdot\|_{\text {hyp }}$ on the invertible sheaf $\omega \mathcal{X} / \mathcal{S}\left(\sigma_{1}+\cdots+\sigma_{n}\right)$. Although $\|\cdot\|_{\text {hyp }}$ is not smooth, its singularities are of some logarithmitc type: it is a pre-log-log hermitian metric in the sense of Burgos-Kramer-Kühn [4, Sec. 7]. The generalizations of the arithmetic intersection theory by Bost [3], Kühn [24] and Burgos-Kramer-Kühn [4] allow one to attach several arithmetic invariants to the metrized invertible sheaf $\omega \mathcal{X} / \mathcal{S}\left(\sigma_{1}+\cdots+\sigma_{n}\right)_{\text {hyp }}$. For instance, if $\mathcal{X}$ is regular, there is a first arithmetic Chern class $\widehat{\mathrm{c}}_{1}\left(\omega \mathcal{X} / \mathcal{S}\left(\sigma_{1}+\cdots+\sigma_{n}\right)_{\text {hyp }}\right)$ in a pre-log-log arithmetic Chow group $\widehat{\mathrm{CH}}{ }_{\text {pre }}^{1}(\mathcal{X})$ [4, Sec. 7]. In general, and if $\mathcal{O}$ is the ring of integers of a number field, there is an arithmetic self-intersection number $\left(\omega \mathcal{X} / \mathcal{S}\left(\sigma_{1}+\cdots+\sigma_{n}\right)_{\text {hyp }}\right)^{2} \in \mathbb{R}$.

In this article we pursue our investigations on an arithmetic Riemann-Roch theorem for pointed stable curves, initiated in [12]. For the sake of simplicity, assume $\mathcal{X}$ is regular. In [12] we exhibited a relation between $\widehat{c}_{1}\left(\omega \mathcal{X} / \mathcal{S}\left(\sigma_{1}+\cdots+\sigma_{n}\right)_{\text {hyp }}\right)^{2} \in \widehat{\mathrm{CH}}_{\text {pre }}^{2}(\mathcal{X})$ and $\lambda(\omega \mathcal{X} / \mathcal{S})_{Q}=\left(\lambda(\omega \mathcal{X} / \mathcal{S}),\|\cdot\|_{Q}\right)$. Here $\|\cdot\|_{Q}$ is a suitable Quillen type metric on $\lambda(\omega \mathcal{X} / \mathcal{S}):=\operatorname{det} R \pi_{*} \omega \mathcal{X} / \mathcal{S}$. Its definition involves the special values at 1 of the derivatives of the Selberg zeta functions $Z\left(\mathcal{U}_{\sigma}(\mathbb{C}), s\right), \sigma \in \Sigma$. A special feature of our formula is the appearance of an arithmetic counterpart $\psi_{W}$ of the so called psi class on $\overline{\mathcal{M}}_{g, n}$. This class detects the continuous spectrum in the resolution of the hyperbolic laplacian on $\mathcal{U}_{\infty}$ (provided that $n>0$ ). Its underlying hermitian structure is Wolpert's renormalization of the hyperbolic metric at the cusps ([37, Def.1] and Definition 4.1 below). In the present work we introduce a Quillen type metric on the determinant $\lambda\left(\omega_{\mathcal{X} / \mathcal{S}}^{k+1}\left(k \sigma_{1}+\cdots+k \sigma_{n}\right)\right)$, for every integer $k \geq 0$ (Definition 4.2). If $k \geq 1$, this metric is built up with the special values $Z\left(\mathcal{U}_{\sigma}(\mathbb{C}), k+1\right), \sigma \in \Sigma$, and the natural $L^{2}$ pairings on $H^{0}\left(\mathcal{X}, \omega_{\mathcal{X} / \mathcal{S}}^{k+1}\left(k \sigma_{1}+\cdots+k \sigma_{n}\right)\right) \otimes_{\sigma} \mathbb{C}, \sigma \in \Sigma$. We relate $\lambda\left(\omega_{\mathcal{X} / \mathcal{S}}^{k+1}\left(k \sigma_{1}+\cdots+k \sigma_{n}\right)\right)_{Q}$ and $\widehat{\mathrm{c}}_{1}\left(\omega \mathcal{X} / \mathcal{S}\left(\sigma_{1}+\cdots+\sigma_{n}\right)\right)^{2}$ through an arithmetic Riemann-Roch formula. Let $\mathcal{O}(C(g, n))$ be the trivial line bundle $\mathcal{O}$ together with the metric $C(g, n)|\cdot|$, where $|\cdot|$ is the absolute value, $C(g, n)=\exp \left((2 g-2+n)\left(\frac{\zeta^{\prime}(-1)}{\zeta(-1)}+\frac{1}{2}\right)\right)$ and $\zeta$ is the Riemann zeta function. The main theorem can be stated as follows.

Theorem A. Let $\left(\mathcal{O}, \Sigma, F_{\infty}\right)$ be an arithmetic ring of Krull dimension at most 1 . Let $\left(\pi: \mathcal{X} \rightarrow \mathcal{S} ; \sigma_{1}, \ldots, \sigma_{n}\right)$ be a generically smooth pointed stable curve of genus $g$. Write $\Delta \mathcal{X} / \mathcal{S} \in \widehat{\mathrm{CH}}^{1}(\mathcal{S})$ for the class of the discriminant of $\pi{ }^{1}$

(i) If $\mathcal{X}$ is regular, then the identity

$$
\begin{aligned}
& 12 \widehat{\mathrm{c}}_{1}\left(\lambda\left(\omega_{\mathcal{X} / \mathcal{S}}^{k+1}\left(k \sigma_{1}+\cdots+k \sigma_{n}\right)\right)_{Q}\right)-\Delta \mathcal{X} / \mathcal{S}+\widehat{\mathrm{c}}_{1}\left(\psi_{W}\right) \\
& \left.=\left(6 k^{2}+6 k+1\right) \pi_{*} \widehat{\mathrm{c}}_{1}\left(\omega \mathcal{X} / \mathcal{S}\left(\sigma_{1}+\cdots+\sigma_{n}\right)_{\text {hyp }}\right)^{2}\right)+\widehat{\mathrm{c}}_{1}(\mathcal{O}(C(g, n)))
\end{aligned}
$$

holds in the arithmetic Chow group $\widehat{\mathrm{CH}}^{1}(\mathcal{S})$.

\footnotetext{
1 The symbol - in this last group is missing in the statement of [12, Thm. A]. The discriminant of $\pi$ is the pull-back of $\mathcal{O}\left(\partial \mathcal{M}_{g, n}\right)$ by the classifying map $\mathcal{S} \rightarrow \overline{\mathcal{M}}_{g, n}$.
} 
(ii) If $\mathcal{O}$ is the ring of integers of a number field and $\mathcal{X}$ is generically smooth, then there is an equality of real numbers

$$
\begin{array}{r}
12 \widehat{\operatorname{deg} \widehat{\mathbf{c}}_{1}}\left(\lambda\left(\omega_{\mathcal{X} / \mathcal{S}}^{k+1}\left(k \sigma_{1}+\cdots+k \sigma_{n}\right)\right)_{Q}\right)-\widehat{\operatorname{deg}} \Delta \mathcal{X} / \mathcal{S}+\widehat{\operatorname{deg}} \widehat{\mathbf{c}}_{1}\left(\psi_{W}\right) \\
\quad=\left(6 k^{2}+6 k+1\right)\left(\omega_{\mathcal{X} / \mathcal{S}}\left(\sigma_{1}+\cdots+\sigma_{n}\right)_{\mathrm{hyp}}\right)^{2}+\widehat{\operatorname{deg}} \widehat{\mathbf{c}}_{1}(\mathcal{O}(C(g, n))) .
\end{array}
$$

Notice that our hypotheses are beyond the reach of the arithmetic Riemann-Roch theorem of Gillet-Soulé [15].

The proof of Theorem A relies on a more general statement: a metrized version of a Mumford type isomorphism on $\mathcal{M}_{g, n}$ (Theorem 6.1). The techniques combine the geometry of the boundary stack $\partial \mathcal{M}_{g+n, 0}$ - through Knudsen's clutching morphisms-and the behavior of the Selberg zeta function (Theorem 5.4) and the $L^{2}$ metric (Theorem 5.6) in suitable degenerating families of Riemann surfaces. Wolpert's pinching expansion for the family hyperbolic metric [36, Exp. 4.2] proves to be a key result in the necessary explicit computations. As a remarkable outcome, Theorem 6.1 implies the Takhtajan-Zograf local index formula for pointed stable curves ([33]-[34] and Corollary 6.5 below).

In arithmetic applications a weaker form of Theorem A may be enough: an arithmetic Hilbert-Samuel type formula. As an advantage, it provides a geometric interpretation of the arithmetic self-intersection number $\left(\omega \mathcal{X} / \mathcal{S}\left(\sigma_{1}+\cdots+\sigma_{n}\right)_{\text {hyp }}\right)^{2}$.

Theorem B. Let $K$ be a number field, $\mathcal{O}_{K}$ its ring of integers and $\mathcal{S}=\operatorname{Spec} \mathcal{O}_{K}$. Let $\left(\pi: \mathcal{X} \rightarrow \mathcal{S} ; \sigma_{1}, \ldots, \sigma_{n}\right)$ be a pointed stable curve with generic fiber $\mathcal{X}_{K}$ smooth. Then there is an asymptotic expansion

$$
\widehat{\operatorname{deg}} \widehat{\mathbf{c}}_{1}\left(\lambda\left(\omega_{\mathcal{X} / \mathcal{S}}^{k+1}\left(k \sigma_{1}+\cdots+k \sigma_{n}\right)\right)_{L^{2}}\right)=\frac{k^{2}}{2}\left(\omega \mathcal{X} / \mathcal{S}\left(\sigma_{1}+\cdots+\sigma_{n}\right)_{\mathrm{hyp}}\right)^{2}+\mathcal{O}(k \log k) .
$$

In particular, the following limit formula holds:

$$
\lim _{k \rightarrow+\infty} \frac{2}{k^{2}} \widehat{\operatorname{deg}} \widehat{\mathrm{c}}_{1}\left(\lambda\left(\omega_{\mathcal{X} / \mathcal{S}}^{k+1}\left(k \sigma_{1}+\cdots+k \sigma_{n}\right)\right)_{L^{2}}\right)=\left(\omega \mathcal{X} / \mathcal{S}\left(\sigma_{1}+\cdots+\sigma_{n}\right)_{\mathrm{hyp}}\right)^{2} .
$$

Notice that the statement of Theorem B does not involve the Quillen metrics, but only the $L^{2}$ metrics on the determinant lines. By means of the uniformization theorem, these norms can be constructed from the Petersson metrics on suitable spaces of automorphic cusp forms.

As an application of Theorem A, we show that the height of points on $\overline{\mathbf{M}}_{0, n}=$ $\overline{\mathcal{M}}_{0, n} /$ isomorphism with respect to the arithmetic $p$ si bundle $\psi_{W}$ satisfies the usual Northcott finiteness property. Also we use Theorem B to understand the first successive minima of the hermitian bundles $\left(H^{0}\left(\mathcal{X}, \omega_{\mathcal{X} / \mathcal{S}}^{k+1}\left(k \sigma_{1}+\cdots+k \sigma_{n}\right)\right),\|\cdot\|_{\text {Pet }}\right), k \geq 0$, for a pointed stable curve of genus 0 and $\|\cdot\|$ Pet the Petersson norm (Section 7).

We would like to stress that the techniques employed in this article do not allow dealing with hermitian vector bundles more general than $\omega_{\mathcal{X} / \mathcal{S}}^{k+1}\left(k \sigma_{1}+\cdots+k \sigma_{n}\right)_{\text {hyp }}$. This is mainly due to the use of moduli spaces of curves and-implicitly-the Selberg trace formula to express the determinant of the hyperbolic laplacian in terms of the Selberg zeta function. 
We briefly review the structure of this paper. In Section 4 we compile most of the normalizations and notations that hold throughout the article. In Section 2 we study the interplay between the adjunction isomorphism and Knudsen's clutching operation. The results obtained are applied in Section 3, where we recall the definition of the tautological invertible sheaves on $\overline{\mathcal{M}}_{g, n}$ and prove relations between them. Section 5 is devoted to the degeneracy of the family Quillen metric. Preliminaries on the continuity properties of the Selberg zeta function in family are provided. These are combined with a careful study of the degeneracy of the family $L^{2}$ metric, whose only merit is due to Obitsu and Wolpert. Theorem A, Theorem B and the Takhtajan-Zograf local index formula are proven in Section 6. Our results are exemplified through the case of pointed stable curves of genus 0 , in Section 7. Finally, in an appendix we give a proof of the ampleness of the line bundle $\kappa_{0, n}$ on $\overline{\mathbf{M}}_{0, n} \rightarrow$ Spec $\mathbb{Z}$, for the sake of completeness.

\section{Adjunction and clutching morphisms}

\subsection{Adjunction isomorphisms for pointed stable curves}

Let $S$ be a noetherian scheme and $\left(\pi: X \rightarrow S ; \sigma_{1}, \ldots, \sigma_{n}\right)$ an $n$-pointed stable curve of genus $g$ [22, Def. 1.1], with $n \geq 1$. The sections $\sigma_{1}, \ldots, \sigma_{n}$ lie in the smooth locus of $\pi$ and define disjoint relative effective Cartier divisors. We indistinctly write $\sigma_{1}, \ldots, \sigma_{n}$ for the sections or for the Cartier divisors they define.

The theory of determinants of Knudsen-Mumford [23, Ch. I] is applied below. We deal with graded invertible $\mathcal{O}_{S}$-modules of the form $(L, a), a \in \mathbb{Z}$ (loc. cit., p. 20). For two such graded invertible sheaves $(L, a),(M, b)$, the commutativity law $(L, a) \otimes_{\mathcal{O}_{S}}$ $(M, b) \stackrel{\sim}{\rightarrow}(M, b) \otimes \mathcal{O}_{S}(L, a)$ is expressed by the Koszul rule $x \otimes y \mapsto(-1)^{a b} y \otimes x$.

Lemma 2.1. Let $S^{\prime} \rightarrow S$ be a morphism of noetherian schemes and $\left(\pi^{\prime}: X^{\prime} \rightarrow\right.$ $\left.S^{\prime} ; \sigma_{1}^{\prime}, \ldots, \sigma_{n}^{\prime}\right)$ the pointed stable curve deduced from $\left(\pi: X \rightarrow S ; \sigma_{1}, \ldots, \sigma_{n}\right)$ by base change to $S^{\prime}$. For chosen integers $1 \leq j_{1}<\cdots<j_{l} \leq n$, put $\sigma=\sigma_{j_{1}}+\cdots+\sigma_{j_{l}}$ (resp. $\sigma^{\prime}=\sigma_{j_{1}}^{\prime}+\cdots+\sigma_{j_{l}}^{\prime}$ ). Then we have canonical isomorphisms of $\mathcal{O}_{X^{\prime}}$-modules

$$
p^{*}\left(\mathcal{O}_{\sigma}\right) \stackrel{\sim}{\rightarrow} \mathcal{O}_{\sigma^{\prime}}
$$

and

$$
p^{*}\left(\mathcal{O}_{X}(-\sigma) / \mathcal{O}_{X}(-\sigma)^{2}\right) \stackrel{\sim}{\rightarrow} \mathcal{O}_{X^{\prime}}\left(-\sigma^{\prime}\right) / \mathcal{O}_{X^{\prime}}\left(-\sigma^{\prime}\right)^{2}
$$

where $p: X^{\prime}=X \times{ }_{S} S^{\prime} \rightarrow X$ is the first projection.

Proof. Immediate from the flatness of the $\mathcal{O}_{S}$-modules $\mathcal{O}_{\sigma}=\mathcal{O}_{X} / \mathcal{O}_{X}(-\sigma)$ and $\mathcal{O}_{X}(-\sigma) / \mathcal{O}_{X}(-\sigma)^{2}$.

Proposition 2.2. Let $\sigma=\sigma_{j_{1}}+\cdots+\sigma_{j_{l}}$, for chosen indices $1 \leq j_{1}<\cdots<j_{l} \leq n$. Let $k \geq 0$ be an integer. Then there is a short exact sequence

$$
0 \rightarrow \omega_{X / S}^{k+1}(k \sigma) \rightarrow \omega_{X / S}(\sigma)^{k+1} \stackrel{\operatorname{Res}_{\sigma}^{k+1}}{\longrightarrow} \mathcal{O}_{\sigma} \rightarrow 0,
$$

compatible with base change. 
Proof. The exact sequence

$$
0 \rightarrow \omega_{X / S}^{k+1}(k \sigma) \rightarrow \omega_{X / S}(\sigma)^{k+1} \rightarrow\left(\omega_{X / S}(\sigma) \otimes_{X} \mathcal{O}_{\sigma}\right)^{k+1} \rightarrow 0
$$

is compatible with base change. We are thus reduced to constructing a canonical isomorphism

$$
\widetilde{\operatorname{Res}}_{\sigma}: \omega_{X / S}(\sigma) \otimes_{\mathcal{O}_{X}} \mathcal{O}_{\sigma} \stackrel{\sim}{\rightarrow} \mathcal{O}_{\sigma}
$$

compatible with base change. First of all $\sigma_{1}, \ldots, \sigma_{n}$ are disjoint sections of $\pi$ lying in the smooth locus. Therefore the differential $d_{X / S}: \mathcal{O}_{X} \rightarrow \Omega_{X / S}$ [16, Sec. 16.3] induces an isomorphism

$$
\tilde{d}_{X / S}: \mathcal{O}_{X}(-\sigma) / \mathcal{O}_{X}(-\sigma)^{2} \stackrel{\sim}{\rightarrow} \omega_{X / S} \otimes \mathcal{O}_{X} \mathcal{O}_{\sigma}
$$

(see [16, Prop. 17.2.5]). If $S^{\prime} \rightarrow S$ is a morphism of noetherian schemes, then there is a commutative diagram [16, Sec. 16.4]

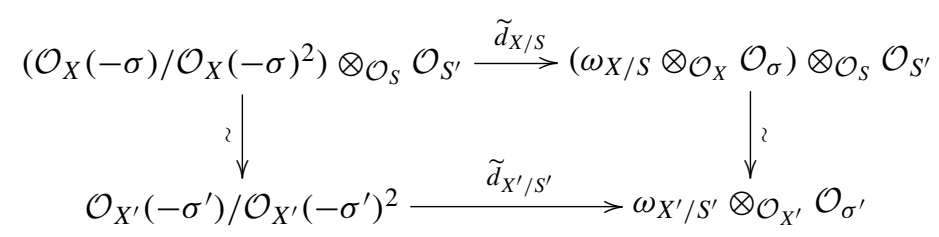

The vertical arrows are isomorphisms due to Lemma 2.1 and the compatibility of the relative dualizing sheaf with base change.

To conclude, we notice the canonical isomorphism

$$
\mathcal{O}_{\sigma} \otimes \mathcal{O}_{X} \mathcal{O}_{X}(-\sigma) \stackrel{\sim}{\rightarrow} \mathcal{O}_{X}(-\sigma) / \mathcal{O}_{X}(-\sigma)^{2}
$$

compatible with base change. The morphism $\widetilde{\operatorname{Res}}_{\sigma}$ is then obtained from $\tilde{d}_{X / S}^{-1}$ by tensoring by $\mathcal{O}_{X}(\sigma)$ and taking (2.2) into account. The compatibility of $\widetilde{\operatorname{Res}} \sigma$ with base change follows from (2.1).

Proposition 2.3. Let $k \geq 1$ be an integer and $\sigma=\sigma_{1}+\cdots+\sigma_{n}$.

(i) There are exact sequences of locally free sheaves on $S$

$$
\begin{aligned}
0 \rightarrow & \pi_{*}\left(\omega_{X / S}^{k+1}\left(k \sigma_{1}+\cdots+k \sigma_{n}\right)\right) \\
& \rightarrow \pi_{*}\left(\omega_{X / S}^{k+1}\left(k \sigma_{1}+\cdots+(k+1) \sigma_{j}+\cdots+k \sigma_{n}\right)\right) \stackrel{\pi_{*} \operatorname{Res}_{\sigma_{j}}^{k+1}}{\longrightarrow} \mathcal{O}_{S} \rightarrow 0
\end{aligned}
$$

and

$$
\begin{aligned}
0 \rightarrow \pi_{*}\left(\omega _ { X / S } ^ { k + 1 } \left(k \sigma_{1}+\right.\right. & \left.\left.\cdots+k \sigma_{n}\right)\right) \\
& \rightarrow \pi_{*}\left(\omega_{X / S}\left(\sigma_{1}+\cdots+\sigma_{n}\right)^{k+1}\right) \stackrel{\pi_{*} \operatorname{Res}_{\sigma}^{k+1}}{\longrightarrow} \mathcal{O}_{S}^{\oplus n} \rightarrow 0,
\end{aligned}
$$

compatible with base change. 
(ii) Let $\delta_{j}: \mathcal{O}_{S} \rightarrow \mathcal{O}_{S}^{\oplus n}$ be the inclusion into the $j$-th factor. There is a natural commutative diagram

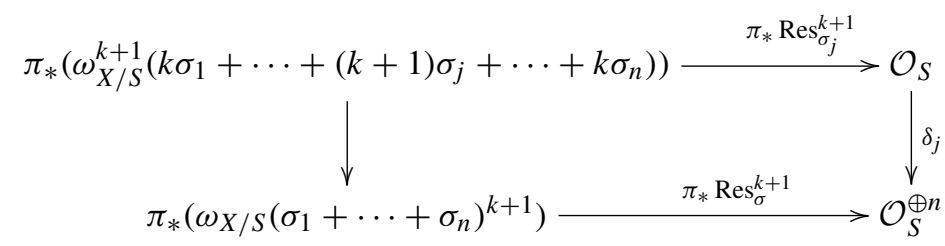

compatible with base change.

Proof. (i) Let us establish the exact sequence (2.4). We leave the proof of (2.3) as an analogous exercise. By Proposition 2.2, we have an exact sequence of coherent sheaves on $X$

$$
0 \rightarrow \omega_{X / S}^{k+1}\left(k \sigma_{1}+\cdots+k \sigma_{n}\right) \rightarrow \omega_{X / S}\left(\sigma_{1}+\cdots+\sigma_{n}\right)^{k+1} \stackrel{\operatorname{Res}_{\sigma}^{k+1}}{\longrightarrow} \mathcal{O}_{\sigma} \rightarrow 0,
$$

commuting with base change. The first two sheaves in (2.5) are flat over $S$ and have vanishing higher direct images $R^{1} \pi_{*}$. Then, from [22, Cor. 1.5], it follows that $\pi_{*}\left(\omega_{X / S}^{k+1}\left(k \sigma_{1}+\cdots+k \sigma_{n}\right)\right)$ and $\pi_{*}\left(\omega_{X / S}\left(\sigma_{1}+\cdots+\sigma_{n}\right)^{k+1}\right)$ are locally free and commute with base change. Furthermore $\pi_{*} \mathcal{O}_{\sigma}=\mathcal{O}_{S}^{\oplus n}$. Therefore, for any morphism $p: S^{\prime} \rightarrow S$ of noetherian schemes, we have a commutative diagram of exact sequences

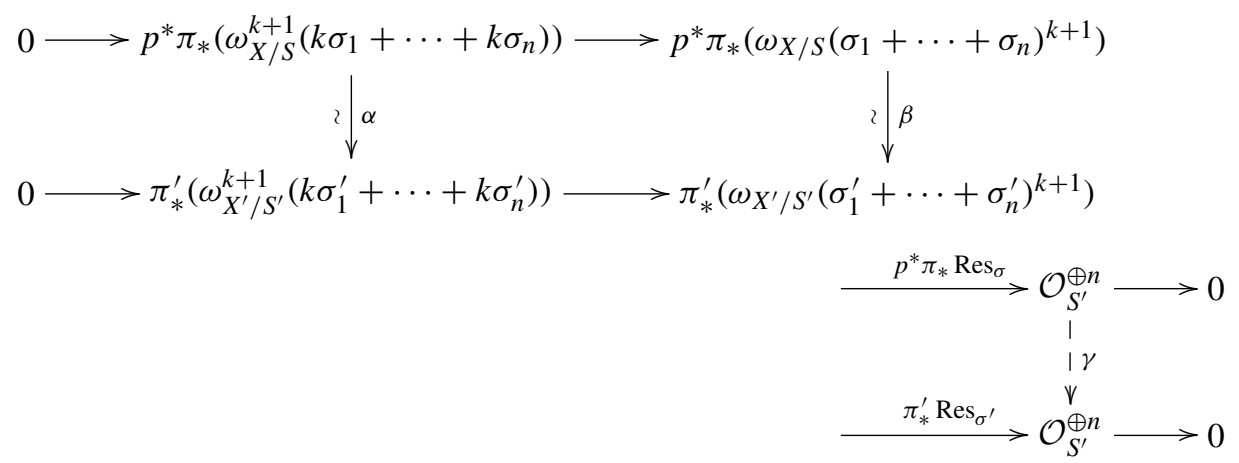

Observe that the vertical arrow $\gamma$ is completely determined by $\alpha$ and $\beta$. In addition, by the five lemma, $\gamma$ is an isomorphism. This completes the proof of the first item.

(ii) The second item is clear from the definition of $\operatorname{Res}_{\sigma_{j}}^{k+1}$ and $\operatorname{Res}_{\sigma}^{k+1}$.

Corollary 2.4. Let $k \geq 0$ be an integer. There are canonical isomorphisms of graded invertible sheaves on $S$

$$
\begin{aligned}
\operatorname{det} R \pi_{*}\left(\omega_{X / S}^{k+1}\left(k \sigma_{1}+(k+1) \sigma_{j}+\cdots+k \sigma_{n}\right)\right) & \\
& \stackrel{\sim}{\rightarrow} \operatorname{det} R \pi_{*}\left(\omega_{X / S}^{k+1}\left(k \sigma_{1}+\cdots+k \sigma_{n}\right)\right) \otimes_{\mathcal{O}_{S}}\left(\mathcal{O}_{S}, 1\right)
\end{aligned}
$$


and

$$
\begin{aligned}
\operatorname{det} R \pi_{*}\left(\omega _ { X / S } \left(\sigma_{1}+\cdots\right.\right. & \left.\left.+\sigma_{n}\right)^{k+1}\right) \\
& \stackrel{\sim}{\rightarrow} \operatorname{det} R \pi_{*}\left(\omega_{X / S}^{k+1}\left(k \sigma_{1}+\cdots+k \sigma_{n}\right)\right) \otimes_{\mathcal{O}_{S}}\left(\mathcal{O}_{S}, n\right),
\end{aligned}
$$

compatible with base change.

Proof. For $k \geq 1$, the corollary already follows from Proposition 2.3 and the theory of determinants of Knudsen-Mumford [23, Ch. I]. The case $k=0$ is individually handled. For the proof of (2.7), first of all Proposition 2.2 provides an exact sequence

$$
0 \rightarrow \omega_{X / S} \rightarrow \omega_{X / S}\left(\sigma_{1}+\cdots+\sigma_{n}\right) \stackrel{\operatorname{Res}_{\sigma}}{\longrightarrow} \mathcal{O}_{\sigma} \rightarrow 0
$$

By [23, Ch. I, p. 46], attached to (2.8) there is a canonical isomorphism of graded invertible sheaves

$$
\operatorname{det} R \pi_{*} \omega_{X / S}\left(\sigma_{1}+\cdots+\sigma_{n}\right) \stackrel{\sim}{\rightarrow}\left(\operatorname{det} R \pi_{*} \omega_{X / S}\right) \otimes \mathcal{O}_{S}\left(\operatorname{det} R \pi_{*} \mathcal{O}_{\sigma}\right),
$$

compatible with base change. Moreover $\operatorname{det} R \pi_{*} \mathcal{O}_{\sigma}=\left(\mathcal{O}_{S}, n\right)$, canonically and commuting with base change. The claim follows. The argument for (2.6) is analogous.

Remark 2.5. (i) The preceding considerations can be effected in the category of complex analytic spaces. The previous results are then compatible with the analytification functor from the category of schemes of finite type over $\mathbb{C}$ to the category of complex analytic spaces.

(ii) Let $\left(\pi: X \rightarrow \operatorname{Spec} \mathbb{C} ; \sigma_{1}, \ldots, \sigma_{n}\right.$ ) be a pointed stable curve. By Chow's lemma we may identify $X$ with its associated complex analytic curve $X^{\text {an }}$. For every $j=1, \ldots, n$, let $u_{j}$ be an analytic coordinate in a neighborhood of $\sigma_{j}$, with $u_{j}\left(\sigma_{j}\right)=0$. Consider $\theta \in H^{0}\left(X, \omega_{X}\left(\sigma_{1}+\cdots+\sigma_{n}\right)^{k+1}\right)$ and express $\theta=\theta_{j}\left(u_{j}\right)\left(d u_{j} / u_{j}\right)^{k+1}$ in the coordinate $u_{j}$, with $\theta_{j}$ holomorphic at 0 . Then, for $\sigma=\sigma_{1}+\cdots+\sigma_{n}$,

$$
\pi_{*} \operatorname{Res}_{\sigma}^{k+1} \theta=\left(\theta_{1}(0), \ldots, \theta_{n}(0)\right) .
$$

Observe that-as expected-this is coordinate independent. The analogous expression for $\pi_{*} \operatorname{Res}_{\sigma_{j}}^{k+1}$ is also true.

\subsection{Clutching morphisms and determinants}

Let $\left(\pi_{1}: X_{1} \rightarrow S ; \sigma_{1}, \ldots, \sigma_{n+1}\right)$ and $\left(\pi_{2}: X_{2} \rightarrow S ; \tau_{1}, \ldots, \tau_{m+1}\right)$ be two pointed stable curves over a noetherian scheme $S$. Knudsen's clutching construction [22, Th. 3.4 and Def. 3.6] applied to the prestable curve $X_{1} \sqcup X_{2} \rightarrow S$, together with the sections $\sigma_{n+1}, \tau_{m+1}$, produces a new prestable curve $\pi: X \rightarrow S$. As a topological space, $X$ is the quotient of $X_{1} \sqcup X_{2}$ under the equivalence relation $\sigma_{n+1} \sim \tau_{m+1}$. The sections $\sigma_{1}, \ldots, \sigma_{n}$, $\tau_{1}, \ldots, \tau_{m}$ define sections of $\pi$. The tuple $\left(\pi: X \rightarrow S ; \sigma_{1}, \ldots, \sigma_{n}, \tau_{1}, \ldots, \tau_{m}\right)$ is a pointed stable curve. 
Proposition 2.6. Let $k \geq 1$ be an integer. Suppose that $S$ is integral. Then there exists a canonical isomorphism of graded invertible sheaves

$$
\begin{aligned}
& \operatorname{det} R \pi_{*} \omega_{X / S}^{k+1}\left(k \sigma_{1}\right.\left.+\cdots+k \sigma_{n}+k \tau_{1}+\cdots+k \tau_{m}\right) \\
& \stackrel{\sim}{\rightarrow}\left(\operatorname{det} R \pi_{1 *} \omega_{X_{1} / S}^{k+1}\left(k \sigma_{1}+\cdots+k \sigma_{n+1}\right)\right) \\
& \otimes_{\mathcal{O}_{S}}\left(\operatorname{det} R \pi_{2 *} \omega_{X_{2} / S}^{k+1}\left(k \tau_{1}+\cdots+k \tau_{m+1}\right)\right) \otimes_{\mathcal{O}_{S}}\left(\mathcal{O}_{S}, 1\right)
\end{aligned}
$$

compatible with base change.

The proof of the proposition will reduce to the construction described below.

Construction 2.7 (Patching pluri-canonical forms). (i) By [22, Th. 3.4], $X$ comes equipped with finite morphisms of $S$-schemes

$$
\iota_{1}: X_{1} \rightarrow X, \quad \iota_{2}: X_{2} \rightarrow X .
$$

By the properties of the relative dualizing sheaf [22, Sec. 1], there is a natural morphism of coherent sheaves

$$
\begin{aligned}
& \omega_{X / S}^{k+1}\left(k \sigma_{1}+\cdots+\right.\left.k \sigma_{n}+k \tau_{1}+\cdots+k \tau_{m}\right) \\
& \rightarrow \iota_{1 *} \omega_{X_{1} / S}^{k+1}\left(k \sigma_{1}+\cdots+k \sigma_{n}+(k+1) \sigma_{n+1}\right) \\
& \quad \oplus \iota_{2 *} \omega_{X_{2} / S}^{k+1}\left(k \tau_{1}+\cdots+k \tau_{n}+(k+1) \tau_{m+1}\right) .
\end{aligned}
$$

If $k \geq 1$, applying $\pi_{*}$ to (2.9), we deduce a morphism of locally free coherent sheaves

$$
\begin{aligned}
& \varphi: \pi_{*} \omega_{X / S}^{k+1}\left(k \sigma_{1}\right.\left.+\cdots+k \sigma_{n}+k \tau_{1}+\cdots+k \tau_{m}\right) \\
& \rightarrow \pi_{1 *} \omega_{X_{1} / S}^{k+1}\left(k \sigma_{1}+\cdots+k \sigma_{n}+(k+1) \sigma_{n+1}\right) \\
& \oplus \pi_{2 *} \omega_{X_{2} / S}^{k+1}\left(k \tau_{1}+\cdots+k \tau_{n}+(k+1) \tau_{m+1}\right) .
\end{aligned}
$$

Notice that the formation of $\varphi$ commutes with base change.

(ii) Replacing $S$ by a smaller Zariski open subset, we may assume that the exact sequences $(2.3)$ for both $\left(\pi_{1} ; \sigma_{1}, \ldots, \sigma_{n+1}\right)$ (with $\left.j=n+1\right)$ and $\left(\pi_{2} ; \tau_{1}, \ldots, \tau_{m+1}\right)$ (with $j=m+1)$ split. Let $\gamma_{1}$ be a lift of $1 \in \mathcal{O}_{S}$ by $\pi_{1 *} \operatorname{Res}_{\sigma_{n+1}}^{k+1}$, and similarly introduce $\gamma_{2}$. We then have non-canonical isomorphisms

$$
\begin{aligned}
\psi_{1}: \pi_{1 *} \omega_{X_{1} / S}^{k+1}\left(k \sigma_{1}+\cdots+k \sigma_{n}\right. & \left.+(k+1) \sigma_{n+1}\right) \\
& \rightarrow \pi_{1 *} \omega_{X_{1} / S}^{k+1}\left(k \sigma_{1}+\cdots+k \sigma_{n+1}\right) \oplus \mathcal{O}_{S}, \\
\theta & \mapsto\left(\theta-\left(\pi_{1 *} \operatorname{Res}_{\sigma_{n+1}}^{k+1} \theta\right) \gamma_{1}, \pi_{1 *} \operatorname{Res}_{\sigma_{n+1}}^{k+1} \theta\right),
\end{aligned}
$$

and an analogously constructed $\psi_{2}$.

(iii) Define $\Phi=\left(\psi_{1} \oplus \psi_{2}\right) \circ \varphi$ and $\widetilde{\Phi}$ the composite of $\Phi$ with the projection onto the factor $\mathcal{O}_{S} \oplus \mathcal{O}_{S}$. We claim that $\operatorname{Im} \widetilde{\Phi}$ is contained in the $\mathcal{O}_{S}$-submodule $\Delta:=$ $\left\{\left(x,(-1)^{k+1} x\right) \in \mathcal{O}_{S} \oplus \mathcal{O}_{S}\right\}$. This is a local assertion: we first reduce to $S$ being an 
affine local noetherian scheme. Secondly, by Nakayama's lemma, we may assume that $S=$ Spec $k$, for $k$ an algebraically closed field. In this case, by the properties of the dualizing sheaf [22, Sec. 1], two forms $\theta_{1} \in H^{0}\left(X_{1}, \omega_{X_{1}}^{k+1}\left(k \sigma_{1}+\cdots k \sigma_{n}+(k+1) \sigma_{n+1}\right)\right)$ and $\theta_{2} \in H^{0}\left(X_{2}, \omega_{X_{2}}^{k+1}\left(k \tau_{1}+\cdots k \tau_{m}+(k+1) \tau_{m+1}\right)\right)$ patch together if, and only if, $\operatorname{Res}_{\sigma_{n+1}} \theta_{1}=(-1)^{k+1} \operatorname{Res}_{\tau_{m+1}} \theta_{2}$. By the very construction of $\Phi$, this completes the proof. Fix the isomorphism $\Delta \stackrel{\sim}{\rightarrow} \mathcal{O}_{S}$ given by the projection onto the first factor. Then, in the definition of $\Phi$, we are allowed to replace $\mathcal{O}_{S} \oplus \mathcal{O}_{S}$ by $\mathcal{O}_{S}$.

Lemma 2.8. Suppose that $S$ is integral. Then $\Phi$ is an isomorphism.

Proof. To check that $\Phi$ is an isomorphism, we may assume that $S$ is an affine local scheme.

Injectivity. Since $S$ is integral, we reduce to the case $S=\operatorname{Spec} k$, where $k$ is an algebraically closed field. The injectivity is then clear by construction of $\Phi$.

Surjectivity. By Nakayama's lemma, we reduce again to the case $S=\operatorname{Spec} k$, where $k$ is an algebraically closed field. The surjectivity of $\Phi$ is then a consequence of the properties of the relative dualizing sheaf [22, Sec. 1].

We are ready to complete the proof of Proposition 2.6.

Proof of Proposition 2.6. Let $\left\{U_{i}\right\}_{i}$ be a finite open covering of $S$ such that over every $U_{i}$ there exists an isomorphism $\Phi_{i}$ as in Construction 2.7. The isomorphisms $\Phi_{i}$ depend on the choices $\left(\gamma_{1}^{(i)}, \gamma_{2}^{(i)}\right)$. Since $S$ is integral, the $\Phi_{i}$ induce isomorphisms

$$
\begin{aligned}
\operatorname{det} \Phi_{i}:\left.\operatorname{det} R \pi_{*} \omega_{X / S}^{k+1}\left(k \sigma_{1}+\cdots+k \sigma_{n}+k \tau_{1}+\cdots+k \tau_{m}\right)\right|_{U_{i}} & \stackrel{\sim}{\rightarrow}\left(\operatorname{det} R \pi_{1 *} \omega_{X_{1} / S}^{k+1}\left(k \sigma_{1}+\cdots+k \sigma_{n+1}\right)\right) \\
& \left.\otimes_{\mathcal{O}_{S}}\left(\operatorname{det} R \pi_{2 *} \omega_{X_{2} / S}^{k+1}\left(k \tau_{1}+\cdots+k \tau_{m+1}\right)\right) \otimes_{\mathcal{O}_{S}}\left(\mathcal{O}_{S}, 1\right)\right|_{U_{i}}
\end{aligned}
$$

One easily checks that the isomorphisms det $\Phi_{i}$ do not depend on the choices $\left(\gamma_{1}^{(i)}, \gamma_{2}^{(i)}\right)$. By construction, the det $\Phi_{i}$ commute with base change. The collection $\left\{\operatorname{det} \Phi_{i}\right\}_{i}$ can be glued into a single canonical isomorphism defined over $S$, compatible with base change. The proposition is proven.

Remark 2.9. The preceding constructions can be made in the category of analytic spaces. They are preserved by the analytification functor from the category of schemes of finite type over $\mathbb{C}$ to the category of analytic spaces.

\section{Tautological line bundles and Mumford isomorphisms}

Let $g, n \geq 0$ be integers with $2 g-2+n>0$ and $\overline{\mathcal{M}}_{g, n} \rightarrow$ Spec $\mathbb{Z}$ the Deligne-Mumford algebraic stack classifying $n$-pointed stable curves of genus $g$ [22]. Let $\pi: \overline{\mathcal{C}}_{g, n} \rightarrow \overline{\mathcal{M}}_{g, n}$ be the universal curve and $\sigma_{1}, \ldots, \sigma_{n}$ the universal sections of $\pi$. We proceed to introduce the tautological line bundles on $\overline{\mathcal{M}}_{g, n}$ and establish relations between them: the Mumford isomorphisms. 
3.1. Tautological line bundles on $\overline{\mathcal{M}}_{g, n}$

Definition 3.1. The tautological line bundles on $\overline{\mathcal{M}}_{g, n}$ are

$$
\begin{aligned}
\lambda_{k+1 ; g, n} & =\operatorname{det} R \pi_{*} \omega_{\overline{\mathcal{C}}_{g, n} / 1}^{k+1} / \overline{\mathcal{M}}_{g, n}\left(k \sigma_{1}+\cdots+k \sigma_{n}\right), \quad k \geq 0, \\
\delta_{g, n} & =\mathcal{O}\left(\partial \mathcal{M}_{g, n}\right), \\
\psi_{g, n}^{(j)} & =\sigma_{j}^{*} \omega_{\overline{\mathcal{C}}_{g, n} / \overline{\mathcal{M}}_{g, n}}, \quad j=1, \ldots, n, \\
\psi_{g, n} & =\bigotimes_{j} \psi_{g, n}^{(j)}, \\
\kappa_{g, n} & =\left\langle\omega_{\overline{\mathcal{C}}_{g, n} / \overline{\mathcal{M}}_{g, n}}\left(\sigma_{1}+\cdots+\sigma_{n}\right), \omega_{\overline{\mathcal{C}}_{g, n} / \overline{\mathcal{M}}_{g, n}}\left(\sigma_{1}+\cdots+\sigma_{n}\right)\right\rangle,
\end{aligned}
$$

where $\langle\cdot, \cdot\rangle$ denotes the Deligne pairing [1, XVIII], [9].

Proposition 3.2. Let $k \geq 0$ be an integer. Then there exists an isomorphism of line bundles on $\overline{\mathcal{M}}_{g, n}$, uniquely determined up to a sign,

$$
\lambda_{k+1 ; g, n} \stackrel{\sim}{\rightarrow} \operatorname{det} R \pi_{*}\left(\omega_{\overline{\mathcal{C}}_{g, n} / \overline{\mathcal{M}}_{g, n}}\left(\sigma_{1}+\cdots+\sigma_{n}\right)^{k+1}\right) .
$$

Proof. The existence of the isomorphism is a reformulation of (2.7) in Corollary 2.4. The uniqueness assertion follows from [12, Cor. 3.2].

Proposition 3.3. Let $\beta: \overline{\mathcal{M}}_{g_{1}, n_{1}+1} \times \overline{\mathcal{M}}_{g_{2}, n_{2}+1} \rightarrow \overline{\mathcal{M}}_{g_{1}+g_{2}, n_{1}+n_{2}}$ be Knudsen's clutching morphism [22, Th. 3.4 and Def. 3.6]. Write $g=g_{1}+g_{2}$ and $n=n_{1}+n_{2}$. Then there are isomorphisms of line bundles on $\overline{\mathcal{M}}_{g_{1}, n_{1}+1} \times \overline{\mathcal{M}}_{g_{2}, n_{2}+1}$, uniquely determined up to a sign,

$$
\begin{aligned}
\beta^{*} \lambda_{k+1 ; g, n} & \stackrel{\sim}{\rightarrow} \lambda_{k+1 ; g_{1}, n_{1}+1} \otimes \lambda_{k+1 ; g_{2}, n_{2}+1}, \quad k \geq 0, \\
\beta^{*} \delta_{g, n} & \stackrel{\sim}{\rightarrow}\left(\delta_{g_{1}, n_{1}+1} \otimes \psi_{g_{1}, n_{1}+1}^{\left(n_{1}+1\right)-1}\right) \otimes\left(\delta_{g_{2}, n_{2}+1} \otimes \psi_{g_{2}, n_{2}+1}^{\left(n_{2}+1\right)-1}\right), \\
\beta^{*} \psi_{g, n} & \stackrel{\sim}{\rightarrow}\left(\psi_{g_{1}, n_{1}+1} \otimes \psi_{g_{1}, n_{1}+1}^{\left(n_{1}+1\right)-1}\right) \otimes\left(\psi_{g_{2}, n_{2}+1} \otimes \psi_{g_{2}, n_{2}+1}^{\left(n_{2}+1\right)-1}\right), \\
\beta^{*} \kappa_{g, n} & \stackrel{\sim}{\rightarrow} \kappa_{g_{1}, n_{1}+1} \otimes \kappa_{g_{2}, n_{2}+1} .
\end{aligned}
$$

Proof. For the isomorphisms (3.2)-(3.3) and (3.1) for $k=0$, we refer to [22, Th. 4.3]. For (3.4) we refer to [12, Prop. 3.7]. The isomorphism (3.1) for $k \geq 1$ is deduced from Proposition 2.6. The uniqueness assertion comes from [12, Cor. 3.2].

Corollary 3.4. Let $\gamma: \overline{\mathcal{M}}_{g, n} \times \overline{\mathcal{M}}_{1,1}^{\times n} \rightarrow \overline{\mathcal{M}}_{g+n, 0}$ be obtained by reiterated applications of clutching morphisms. Then we have isomorphisms, uniquely determined up to a sign,

$$
\begin{aligned}
\gamma^{*} \lambda_{k+1 ; g+n, 0} & \stackrel{\sim}{\rightarrow} \lambda_{k+1 ; g, n} \otimes \lambda_{k+1 ; 1,1}^{\otimes n}, \quad k \geq 0, \\
\gamma^{*} \delta_{g+n, 0} & \stackrel{\sim}{\rightarrow}\left(\delta_{g, n} \otimes \psi_{g, n}^{-1}\right) \otimes\left(\delta_{1,1} \otimes \psi_{1,1}^{-1}\right)^{\otimes n}, \\
\gamma^{*} \kappa_{g+n, 0} & \stackrel{\sim}{\rightarrow} \kappa_{g, n} \otimes \kappa_{1,1}^{\otimes n} .
\end{aligned}
$$

Proof. This is straightforward from Proposition 3.3 and [12, Cor. 3.2]. 


\subsection{Mumford isomorphisms}

Theorem 3.5 (Mumford isomorphism). Let $k \geq 0$ be an integer. There is an isomorphism of invertible sheaves on $\overline{\mathcal{M}}_{g, n}$, uniquely determined up to a sign,

$$
\mathcal{D}_{k+1 ; g, n}: \lambda_{k+1 ; g, n}^{\otimes 12} \otimes \delta_{g, n}^{-1} \otimes \psi_{g, n} \stackrel{\sim}{\rightarrow} \kappa_{g, n}^{\otimes\left(6 k^{2}+6 k+1\right)} .
$$

The proof of the theorem relies on [12, Th. 3.10] and the following lemma.

Lemma 3.6. Let $k \geq 1$ be an integer. There is an isomorphism of invertible sheaves on $\overline{\mathcal{M}}_{g, n}$

$$
\lambda_{k+1 ; g, n} \stackrel{\sim}{\rightarrow} \lambda_{k ; g, n} \otimes \kappa_{g, n}^{\otimes k} .
$$

Proof. We proceed in two steps.

Step 1. We first treat the cases $g=n=1$ or $g \geq 2, n=0$. Let $\mathcal{M}=\overline{\mathcal{M}}_{1,1}$ or $\overline{\mathcal{M}}_{g, 0}$, $\mathcal{C}=\overline{\mathcal{C}}_{1,1}$ or $\overline{\mathcal{C}}_{g, 0}$. Recall the cohomological expression for the Deligne-Weil pairing [32, Exp. II], [26]: given line bundles $L, M$ on $\mathcal{C}$, there is an isomorphism of invertible sheaves

$$
\langle L, M\rangle \simeq \operatorname{det} R \pi_{*}(L \otimes M) \otimes\left(\operatorname{det} R \pi_{*} L\right)^{-1} \otimes\left(\operatorname{det} R \pi_{*} M\right)^{-1} \otimes \operatorname{det} R \pi_{*} \mathcal{O}_{\mathcal{C}} .
$$

If $g \geq 2$ and $n=0$, then (3.9) applied to $L=\omega_{\mathcal{C} / \mathcal{M}}^{\otimes k}, M=\omega_{\mathcal{C} / \mathcal{M}}$ and Serre's duality already prove (3.8). If $g=n=1$, we set $L=\omega_{\mathcal{C} / \mathcal{M}}(\sigma)^{k}$ and $M=\omega_{\mathcal{C} / \mathcal{M}}(\sigma)$. The conclusion follows by combination of (3.9), Proposition 3.2 and Serre's duality.

Step 2. For the general case, introduce the clutching morphism $\gamma: \overline{\mathcal{M}}_{g, n} \times \overline{\mathcal{M}}_{1,1}^{\times n} \rightarrow$ $\overline{\mathcal{M}}_{g+n, 0}$. From Corollary 3.4 and the first step, there are isomorphisms

$$
\lambda_{k+1 ; g, n} \otimes \lambda_{k+1 ; 1,1}^{\bigotimes n} \stackrel{\sim}{\rightarrow}\left(\lambda_{k ; g, n} \otimes \kappa_{g, n}^{\otimes k}\right) \otimes\left(\lambda_{k ; 1,1} \otimes \kappa_{1,1}^{\otimes k}\right)^{\bigotimes n}
$$

and

$$
\operatorname{pr}_{2}^{*} \lambda_{k+1 ; 1,1}^{\bigotimes n} \stackrel{\sim}{\rightarrow} \operatorname{pr}_{2}^{*}\left(\lambda_{k ; 1,1} \otimes \kappa_{1,1}^{\otimes k}\right)^{\bigotimes n},
$$

where $\operatorname{pr}_{2}: \overline{\mathcal{M}}_{g, n} \times \overline{\mathcal{M}}_{1,1}^{\times n} \rightarrow \overline{\mathcal{M}}_{1,1}^{\times n}$ is the projection onto the second factor. The isomorphisms (3.10)-(3.11) together provide an isomorphism

$$
\operatorname{pr}_{1}^{*} \lambda_{k+1 ; g, n} \stackrel{\sim}{\rightarrow} \operatorname{pr}_{1}^{*}\left(\lambda_{k ; g, n} \otimes \kappa_{g, n}^{\otimes k}\right) \text {. }
$$

To conclude, consider the section $\Delta: \operatorname{Spec} \mathbb{Z} \rightarrow \overline{\mathcal{M}}_{1,1}^{\times n}$ induced by $n$ copies of the 1pointed stable curve $\left(\mathbb{P}_{\mathbb{Z}}^{1} /\{0 \sim \infty\} ; 1\right)$. It induces a section $\varphi=$ id $\times \Delta: \overline{\mathcal{M}}_{g, n} \rightarrow$ $\overline{\mathcal{M}}_{g, n} \times \overline{\mathcal{M}}_{1,1}^{\times n}$ over $\overline{\mathcal{M}}_{g, n}$. Pulling back (3.12) by $\varphi$, we obtain the desired isomorphism.

Proof of Theorem 3.5. The proof is by induction. The case $k=0$ is the content of [12, Th. 3.10]. The induction step is achieved by means of Lemma 3.6. Uniqueness up to a sign is ensured by [12, Cor. 3.2].

Corollary 3.7. Let $\gamma: \overline{\mathcal{M}}_{g, n} \times \overline{\mathcal{M}}_{1,1}^{\times n} \rightarrow \overline{\mathcal{M}}_{g+n, 0}$ be a reiterated clutching morphism. Then the isomorphisms $\gamma^{*} \mathcal{D}_{k+1 ; g+n, 0}$ and $\mathcal{D}_{k+1 ; g, n} \otimes \mathcal{D}_{k+1 ; 1,1}^{\bigotimes n}$ are equal up to a sign.

Proof. Combine Theorem 3.5, Corollary 3.4 and [12, Cor. 3.2]. 


\section{Hermitian tautological line bundles}

The main step towards the proof of Theorem A is an extension of Theorem 3.5 to suitable hermitian tautological line bundles. We now introduce the metrics we need and give the definition of the hermitian counterparts of the tautological line bundles.

\subsection{Definition of metrics}

Let $g, n \geq 0$ be integers with $2 g-2+n>0$. We fix $X$ a compact and connected Riemann surface of genus $g$ and $p_{1}, \ldots, p_{n}$ distinct points in $X$. We denote $X^{\circ}=X \backslash\left\{p_{1}, \ldots, p_{n}\right\}$.

Constants. We define the real constants

$$
\begin{aligned}
C(g, n)= & \exp \left((2 g-2+n)\left(\frac{\zeta^{\prime}(-1)}{\zeta(-1)}+\frac{1}{2}\right)\right) \\
E_{1}(g, n)= & 2^{(g+2-n) / 3} \pi^{-n / 2} \\
& \cdot \exp \left((2 g-2+n)\left(2 \zeta^{\prime}(-1)-\frac{1}{4}+\frac{1}{2} \log (2 \pi)\right)\right)
\end{aligned}
$$

and, for every integer $k \geq 1$,

$$
\begin{aligned}
C_{k}= & 2 \zeta^{\prime}(-1)-\left(k+\frac{1}{2}\right)^{2}+\left(k+\frac{1}{2}\right) \log (2 \pi)+\sum_{j=1}^{2 k}\left(j-k-\frac{1}{2}\right) \log j \\
& +\frac{3 k+1}{6} \log 2, \\
E_{k+1}(g, n)= & 2^{-(3 k+2) n / 3}\left((2 k) ! \pi^{2 k+1}\right)^{-n / 2} \exp \left((2 g-2+n) C_{k}\right) .
\end{aligned}
$$

Here $\zeta$ denotes the Riemann zeta function. Notice the relations

$$
\begin{aligned}
C(g+n, 0) & =C(g, n) C(1,1)^{n}, \\
E_{1}(g+n, 0) & =\pi^{n} E_{1}(g, n) E_{1}(1,1)^{n}, \\
E_{k+1}(g+n, 0) & =\left(2^{k+1}(2 k) ! \pi^{2 k+1}\right)^{n} E_{k+1}(g, n) E_{k+1}(1,1)^{n}, \quad k \geq 1 .
\end{aligned}
$$

Hyperbolic and Wolpert metrics. Observe that $X^{\circ}=X \backslash\left\{p_{1}, \ldots, p_{n}\right\}$ admits a complete hyperbolic riemannian metric of constant curvature -1 . Denote it by $d s_{\text {hyp }, X^{\circ}}^{2}$. Via a fuchsian uniformization $X^{\circ} \simeq \Gamma \backslash \mathbb{H}, \Gamma \subset \mathrm{PSL}_{2}(\mathbb{R})$ torsion free, the metric $d s_{\text {hyp }, X^{\circ}}^{2}$ is obtained by descent from the $\Gamma$ invariant Riemann tensor on $\mathbb{H}$

$$
d s_{\text {hyp }, \mathbb{H}}^{2}=\frac{d x^{2}+d y^{2}}{y^{2}}, \quad z=x+i y \in \mathbb{H} .
$$

Associated to $d s_{\text {hyp }, X^{\circ}}^{2}$ there is a hermitian metric on the complex line $T_{X^{\circ}}$, which we write $h_{X^{\circ}}$. It is obtained by descent from the metric $h_{\mathbb{H}}$ on $T_{\mathbb{H}}$ defined by the rule

$$
h_{\mathbb{H}}\left(\frac{\partial}{\partial z}, \frac{\partial}{\partial z}\right)=\frac{1}{2 y^{2}} .
$$


The hermitian metric $h_{X}$ extends to a pre-log-log hermitian metric $\|\cdot\|_{\text {hyp }}$ on $\omega_{X}\left(p_{1}+\cdots+p_{n}\right)\left[11\right.$, Sec. 7.3.2]. The first Chern form of $\omega_{X}\left(p_{1}+\cdots+p_{n}\right)_{\text {hyp }}$, which is defined on $X^{\circ}$, coincides with the normalized Kähler form $\omega$ of $h_{X^{\circ}}$ (curvature -1 condition). The form $\omega$ is locally given by

$$
\omega=\frac{i}{2 \pi} h_{X}\left(\frac{\partial}{\partial z}, \frac{\partial}{\partial z}\right) d z \wedge d \bar{z} .
$$

The volume of $X$ with respect to $\omega$ is $2 g-2+n$.

For every puncture $p_{j}$ there is a conformal coordinate $z$ with $z\left(p_{j}\right)=0$, by means of which a small punctured disc $D^{*}(0, \varepsilon) \subset \mathbb{C}$ with the Poincaré metric

$$
d s_{P}^{2}=\left(\frac{|d z|}{|z| \log |z|}\right)^{2}
$$

isometrically embeds into $\left(X^{\circ}, d s_{\text {hyp, } X^{\circ}}^{2}\right)$. Such a coordinate is unique up to rotation and is called an rs coordinate at the cusp $p_{j}$.

Definition 4.1 (Wolpert metric [37, Def. 1]). Let $z$ be an $r s$ coordinate at the cusp $p_{j}$. The Wolpert metric on the complex line $\omega_{X, p_{j}}$ is defined by

$$
\|d z\|_{W, p_{j}}=1 .
$$

The tensor product $\bigotimes_{j} \omega_{X, p_{j}}$ is equipped with the tensor product of Wolpert metrics, and we write $\|\cdot\|_{W}$ for the resulting metric.

$L^{2}$ metrics. We introduce the $L^{2}$ metrics on the determinant line bundles $\lambda\left(\omega_{X}^{k+1}\left(k p_{1}+\right.\right.$ $\left.\left.\cdots+k p_{n}\right)\right)$. The cases $k=0$ and $k \geq 1$ need to be distinguished.

The complex vector space $\mathcal{C}^{\infty}\left(X, \omega_{X}\right)\left(\supset H^{0}\left(X, \omega_{X}\right)\right)$ is equipped with the nondegenerate hermitian form

$$
\langle\alpha, \beta\rangle_{0}=\frac{i}{2 \pi} \int_{X} \alpha \wedge \bar{\beta}
$$

The space $H^{1}\left(X, \omega_{X}\right)^{\vee}$ is canonically isomorphic to $H^{0}\left(X, \mathcal{O}_{X}\right)=\mathbb{C}$ via the analytic Serre duality. Since $\omega$ is integrable, the $L^{2}$ metric on $\mathcal{C}^{\infty}\left(X, \mathcal{O}_{X}\right)\left(\supset H^{0}\left(X, \mathcal{O}_{X}\right)\right)$ with respect to $h_{X^{\circ}}$ is well defined. If $\mathbf{1}$ is the function with constant value 1 , then

$$
\langle\mathbf{1}, \mathbf{1}\rangle_{1}=\int_{X} \omega=2 g-2+n .
$$

The complex line $\lambda\left(\omega_{X}\right)=\operatorname{det} H^{0}\left(X, \omega_{X}\right) \otimes \operatorname{det} H^{1}\left(X, \omega_{X}\right)^{-1}$ is endowed with the determinant metric build up from $\langle\cdot, \cdot\rangle_{0}$ and $\langle\cdot, \cdot\rangle_{1}$. We denote it by $\|\cdot\|_{L^{2}}$.

Let $k \geq 1$ be an integer. Notice that $H^{1}\left(X, \omega_{X}^{k+1}\left(k p_{1}+\cdots+k p_{n}\right)\right)=0$. Hence

$$
\begin{aligned}
\lambda\left(\omega_{X}^{k+1}\left(k p_{1}+\cdots+k p_{n}\right)\right): & =\operatorname{det} R \Gamma\left(X, \omega_{X}^{k+1}\left(k p_{1}+\cdots+k p_{n}\right)\right) \\
& =\operatorname{det} H^{0}\left(X, \omega_{X}^{k+1}\left(k p_{1}+\cdots+k p_{n}\right)\right) .
\end{aligned}
$$


The line bundle $\omega_{X}^{k+1}\left(k p_{1}+\cdots+k p_{n}\right)$ inherits a-singular-hermitian structure from $\omega_{X}\left(p_{1}+\cdots+p_{n}\right)_{\text {hyp }}^{k+1}$. Denote it by $\langle\cdot, \cdot\rangle_{\text {hyp }}$. For $\alpha, \beta \in H^{0}\left(X, \omega_{X}^{k+1}\left(k p_{1}+\cdots+k p_{n}\right)\right)$, the integral

$$
\langle\alpha, \beta\rangle_{L^{2}}:=\int_{X}\langle\alpha, \beta\rangle_{\text {hyp }} \omega
$$

is absolutely convergent. ${ }^{2}$ Indeed, because $X$ is compact, it is enough to observe that in an $r s$ coordinate $z$ at a cusp $p_{j}$, there is an estimate

$$
|\langle\alpha, \beta\rangle \omega|=O\left((\log |z|)^{2 k}|d z \wedge d \bar{z}|\right),
$$

which is a locally integrable form. Therefore this rule defines a hermitian metric on $H^{0}\left(X, \omega_{X}^{k+1}\left(k p_{1}+\cdots+k p_{n}\right)\right)$. We write $\|\cdot\|_{L^{2}}$ for the determinant metric on $\lambda\left(\omega_{X}^{k+1}\left(k p_{1}+\cdots+k p_{n}\right)\right)$.

Selberg zeta function and Quillen metric. We next recall the definition of the Selberg zeta function of $X^{\circ}$ (see [19]). For every real $l>0$ the function

$$
Z_{l}(s)=\prod_{k=0}^{\infty}\left(1-e^{-(s+k) l}\right)^{2}
$$

is holomorphic in $\operatorname{Re} s>0$. In a first step, the Selberg zeta function of $X^{\circ}$ is defined by the absolutely convergent product

$$
Z\left(X^{\circ}, s\right)=\prod_{\gamma} Z_{l(\gamma)}(s), \quad \operatorname{Re} s>1,
$$

running over the primitive closed non-oriented geodesics $\gamma$ of the hyperbolic surface $\left(X^{\circ}, d s_{\text {hyp }, X^{\circ}}^{2}\right)$, of length $l(\gamma)$. Then one shows that $Z\left(X^{\circ}, s\right)$ extends to a meromorphic function on $\mathbb{C}$, with a simple zero at $s=1$.

Definition 4.2 (Quillen metric). Let $k \geq 0$ be an integer. We define the Quillen metric on $\lambda\left(\omega_{X}^{k+1}\left(k p_{1}+\cdots+k p_{n}\right)\right)$, attached to the hyperbolic metric on $X^{\circ}$, to be

$$
\|\cdot\|_{Q}= \begin{cases}\left(E_{1}(g, n) Z^{\prime}\left(X^{\circ}, 1\right)\right)^{-1 / 2}\|\cdot\|_{L^{2}} & \text { if } k=0 \\ \left(E_{k+1}(g, n) Z\left(X^{\circ}, k+1\right)\right)^{-1 / 2}\|\cdot\|_{L^{2}} & \text { if } k \geq 1 .\end{cases}
$$

When $n=0$ and hence $X=X^{\circ}$ is compact hyperbolic of genus $g \geq 2$, a result of Sarnak [28] completed by [8, Cor. 1.12] guarantees that $\|\cdot\|_{Q}$ equals the usual Quillen metric on $\lambda\left(\omega_{X \text {,hyp }}^{k+1}\right)$ with respect to the hyperbolic hermitian structure on $T_{X}$ (see also the proof of Proposition 6.2).

Notice the apparent discrepancy between the definition of the Quillen metric for $k=0$ and for $k \geq 1$ (recall the definition of $E_{1}(g, n)$ and $E_{k+1}(g, n)$ for $k \geq 1$ ). The difference actually stems from the fact $H^{1}\left(X, \omega_{X}\right) \neq 0$, while $H^{1}\left(X, \omega_{X}^{k+1}\left(k p_{1}+\cdots+k p_{n}\right)\right)=0$ for $k \geq 1$.

\footnotetext{
2 However this fails to be true for the bigger space $H^{0}\left(X, \omega_{X}\left(\sigma_{1}+\cdots+\sigma_{n}\right)^{k+1}\right)$.
} 
First tautological metric. The first tautological metric on the Deligne self-pairing $\left\langle\omega_{X}\left(p_{1}+\cdots+p_{n}\right), \omega_{X}\left(p_{1}+\cdots+p_{n}\right)\right\rangle$ is the hermitian structure defined in [12, Sec. 4]. We will denote it by $\|\cdot\|_{F T}$.

\subsection{Hermitian tautological line bundles}

Let $(\cdot)^{\text {an }}$ be the analytification functor from the category of algebraic stacks of finite type over Spec $\mathbb{Z}$ to the category of analytic stacks. We employ the same notation for the functor sending coherent sheaves on an algebraic stack $F$ of finite type over Spec $\mathbb{Z}$ to analytic coherent sheaves on $F^{\text {an }}$. For instance, applying this functor to $\mathcal{M}_{g, n}$ and $\overline{\mathcal{M}}_{g, n}$, we obtain the analytic stack $\mathcal{M}_{g, n}^{\text {an }}$ of $n$-punctured Riemann surfaces of genus $g$ and its DeligneMumford stable compactification $\overline{\mathcal{M}}_{g, n}^{\text {an }}$. The analytic line bundles $\lambda_{k+1 ; g, n}^{\text {an }}, \psi_{g, n}^{(j) \text { an }}, \psi_{g, n}^{\text {an }}$, $\kappa_{g, n}^{\mathrm{an}}$ may be enriched with pointwise hermitian structures defined on $\mathcal{M}_{g, n}^{\mathrm{an}}$. This amounts to giving, for every $n$-pointed Riemann surface of genus $g,\left(X ; p_{1}, \ldots, p_{n}\right)$, hermitian structures on $\lambda\left(\omega_{X}^{k+1}\left(k p_{1}+\cdots+k p_{n}\right)\right), \omega_{X, p_{j}}, \bigotimes_{j} \omega_{X, p_{j}}$ and $\left\langle\omega_{X}\left(p_{1}+\cdots+p_{n}\right)\right.$, $\left.\omega_{X}\left(p_{1}+\cdots+p_{n}\right)\right\rangle$. We choose the hermitian metrics introduced in Subsection 4.1 and use the same notations for them. We thus obtain hermitian line bundles on $\overline{\mathcal{M}}_{g, n}$

$$
\begin{aligned}
& \lambda_{k+1 ; g, n ; L^{2}}=\left(\lambda_{k+1 ; g, n},\|\cdot\|_{L^{2}}\right), \quad \psi_{g, n ; W}=\left(\psi_{g, n},\|\cdot\|_{W}\right), \\
& \lambda_{k+1 ; g, n ; Q}=\left(\lambda_{k+1 ; g, n},\|\cdot\|_{Q}\right), \quad \kappa_{g, n ; F T}=\left(\kappa_{g, n},\|\cdot\|_{F T}\right) .
\end{aligned}
$$

whose metrics are defined on $\mathcal{M}_{g, n}^{\text {an }}$. In addition, it will be convenient to consider the trivial metric on $\delta_{g, n}^{\text {an }}$, giving to the trivial section 1 the constant value 1 . Observe that this metric makes sense only on $\mathcal{M}_{g, n}^{\mathrm{an}}$. We will write $\delta_{g, n ; T}=\left(\delta_{g, n},\|\cdot\|_{T}\right)$ for the corresponding hermitian line bundle. In particular, the restriction $\left.\delta_{g, n ; T}\right|_{\mathcal{M}_{g, n}}$ is the trivial hermitian line bundle on $\mathcal{M}_{g, n}$. Finally, for every real constant $C>0$, the notation $\mathcal{O}(C)$ stands for the trivial line bundle $\mathcal{O}$ with the hermitian metric $C|\cdot|$, where $|\cdot|$ is the usual absolute value.

We end this section by recalling a continuity property of the first tautological metric $\|\cdot\|_{F T}$.

Theorem 4.3 ([12, Lemma 4.6 and Cor. 4.8]). The first tautological metric on $\kappa_{g, n}$ uniquely extends to a continuous metric on $\overline{\mathcal{M}}_{g, n}$. Furthermore, if $\gamma: \overline{\mathcal{M}}_{g, n} \times \overline{\mathcal{M}}_{1,1}^{\times n} \rightarrow$ $\overline{\mathcal{M}}_{g+n, 0}$ is a reiterated clutching morphism, then the isomorphism (3.7) $\gamma^{*} \kappa_{g+n, 0} \stackrel{\sim}{\rightarrow}$ $\kappa_{g, n} \otimes \kappa_{1,1}^{\otimes n}$ becomes an isometry for the extended first tautological metrics.

\section{Degeneracy of the Quillen metric}

Let $g, n \geq 0$ be integers with $2 g-2+n>0$. Let $\gamma: \overline{\mathcal{M}}_{g, n} \times \overline{\mathcal{M}}_{1,1} \rightarrow \overline{\mathcal{M}}_{g+n, 0}$ be a reiterated clutching morphism. The object of study of this section is the behavior of the Quillen metric on $\lambda_{k+1 ; g+n, 0}^{\text {an }}$ near the image of $\gamma^{\text {an }}$ in $\overline{\mathcal{M}}_{g+n, 0}^{\text {an }}$. Before the statement of the main theorem we introduce some notations that will prevail until the end. 
Let $\left(X ; a_{1}, \ldots, a_{n}\right)$ be a smooth $n$-pointed stable complex curve of genus $g$, and $\left(T_{1} ; b_{1}\right), \ldots,\left(T_{n} ; b_{n}\right) n$ smooth 1 -pointed stable complex curves of genus 1 . These data define complex valued points $P \in \overline{\mathcal{M}}_{g, n}(\mathbb{C}), Q_{1}, \ldots, Q_{n} \in \overline{\mathcal{M}}_{1,1}(\mathbb{C})$. The image of $N=\left(P_{1}, Q_{1}, \ldots, Q_{n}\right)$ under the reiterated clutching morphism $\gamma: \overline{\mathcal{M}}_{g, n} \times \overline{\mathcal{M}}_{1,1}^{\times n} \rightarrow$ $\overline{\mathcal{M}}_{g+n, 0}$ is a complex valued point $R \in \overline{\mathcal{M}}_{g+n, 0}(\mathbb{C})$. Notice that $R$ represents the quotient analytic space

$$
Y=\left(X \sqcup T_{1} \sqcup \cdots \sqcup T_{n}\right) /\left(a_{1} \sim b_{1}, \ldots, a_{n} \sim b_{n}\right) .
$$

The compactness of $Y$ ensures its algebraicity. Following [36, Sec. 2] and [12, Cons. 4.1], we construct an analytic one-parameter small stable deformation of $Y$, to be denoted $\mathfrak{g}: \mathfrak{Z} \rightarrow D=D(0, \varepsilon)$. The fibers $\mathfrak{g}^{-1}(t)=\mathfrak{Z}_{t}$, with $t \neq 0$, are smooth complex compact curves, whereas $Y=\mathfrak{Z}_{0}$ is singular. For every $j=1, \ldots, n$, there are $r s$ coordinates $u_{j}$ at $a_{j}$ and $v_{j}$ at $b_{j}$ such that a neighborhood of $a_{j} \sim b_{j}$ in $\mathfrak{Z}$ is isomorphic to the family $u_{j} v_{j}=t$ fibered over $t$.

Let $k \geq 1$ be an integer. We construct and deform a basis of $H^{0}\left(Y, \omega_{Y}^{k+1}\right)$, according to Proposition 2.2 and Construction 2.7.

Construction 5.1 (Frames of pluri-canonical forms).

(i) Fix bases $\alpha_{1}, \ldots, \alpha_{a}$ of $H^{0}\left(X, \omega_{X}^{k+1}\left(k a_{1}+\cdots+k a_{n}\right)\right)$ and $\beta_{1}^{(j)}, \ldots, \beta_{b}^{(j)}$ of $H^{0}\left(T_{j}, \omega_{T_{j}}^{k+1}\left(k b_{j}\right)\right)$. Notice that $\operatorname{Res}_{a_{i}}^{k+1} \alpha_{j}=0$ for all $i, j$ and $\operatorname{Res}_{b_{j}}^{k+1} \beta_{l}^{(j)}=0$ for all $j, l$. Therefore, extending the forms $\alpha_{i}, \beta_{l}^{(j)}$ by 0 produces global sections of $\omega_{Y}^{k+1}$. We use the same symbols for these extensions.

(ii) For every $j=1, \ldots, n$, choose a form $\gamma_{j} \in H^{0}\left(X, \omega_{X}^{k+1}\left(k a_{1}+\cdots+(k+1) a_{j}+\right.\right.$ $\left.\cdots+k a_{n}\right)$ with $\operatorname{Res}_{a_{j}}^{k+1} \gamma_{j}=1$. Also, let $\phi_{j} \in H^{0}\left(T_{j}, \omega_{T_{j}}\left(b_{j}\right)^{k+1}\right)$ be a form with $\operatorname{Res}_{b_{j}}^{k+1} \phi_{j}=(-1)^{k+1}$. By Construction 2.7 (patching pluri-canonical forms) we can glue the forms $\gamma_{j}, \phi_{j}$ into a global section $\theta_{j}$ of $\omega_{Y}^{k+1}$.

(iii) By reiterated applications of Lemma 2.8, $\alpha_{i}, \beta_{l}^{(j)}, \theta_{k}$ for all $i, j, k, l$ constitute a basis of $H^{0}\left(Y, \omega_{Y}^{k+1}\right)$.

(iv) The sheaf $R \mathfrak{g}_{*} \omega_{\mathfrak{Z} / D}^{k+1}$ is locally free. Hence, after possibly shrinking $D$, we can extend the family of forms $\alpha_{i}, \beta_{l}^{(j)}, \theta_{k}$ to a frame $\alpha_{i}(t), \beta_{l}^{(j)}(t), \theta_{k}(t)$ of $R \mathfrak{g}_{*} \omega_{\mathfrak{Z} / D}^{k+1}$.

Lemma 5.2. Let $\Psi: \gamma^{*} \lambda_{k+1 ; g+n, 0} \stackrel{\sim}{\rightarrow} \lambda_{k+1 ; g, n} \otimes \lambda_{k+1 ; 1,1}^{\otimes n}$ be the isomorphism (3.5). Then $\Psi$ induces an isomorphism

$$
\begin{gathered}
\operatorname{det} H^{0}\left(Y, \omega_{Y}^{k+1}\right) \stackrel{\sim}{\rightarrow} \operatorname{det} H^{0}\left(X, \omega_{X}^{k+1}\left(k a_{1}+\cdots+k a_{n}\right)\right) \otimes \bigotimes_{j=1}^{n} \operatorname{det} H^{0}\left(T_{j}, \omega_{T_{j}}^{k+1}\left(k b_{j}\right)\right), \\
\theta_{1} \wedge \cdots \wedge \theta_{n} \wedge \alpha_{1} \wedge \cdots \wedge \beta_{b}^{(n)} \mapsto \pm \alpha_{1} \wedge \cdots \wedge \alpha_{n} \otimes \beta_{1}^{(1)} \wedge \cdots \otimes \cdots \otimes \cdots \wedge \beta_{b}^{(n)} .
\end{gathered}
$$

Proof. This is easily checked from Construction 2.7 and the proof of Proposition 2.6.

Attached to the family $\mathfrak{g}: \mathfrak{Z} \rightarrow D$, there is a classifying map

$$
\mathcal{C}(\mathfrak{g}): D \rightarrow \overline{\mathcal{M}}_{g+n, 0}^{\text {an }} \text {. }
$$


The line bundle $\operatorname{det} R \mathfrak{g}_{*} \omega_{\mathfrak{Z} / D}^{k+1}=\mathcal{C}(\mathfrak{g})^{*} \lambda_{k+1 ; g+n, 0}^{\text {an }}$ is endowed with the family Quillen metric $\|\cdot\|_{Q}$. This metric is defined and smooth on $D \backslash\{0\}$.

The following statement will allow us to reduce the proof of Theorem A (more generally Theorem 6.1 below) to the known arithmetic Riemann-Roch theorem in the compact case. The proof is detailed in the next subsections.

Theorem 5.3. Let $k \geq 1$ be an integer and $\mathfrak{g}: \mathfrak{Z} \rightarrow D$ a one-dimensional stable deformation of $Y$ as above. Let $\alpha_{i}(t), \beta_{l}^{(j)}(t), \theta_{k}(t)$ be a frame of $R \mathfrak{g}_{*} \omega_{\mathfrak{Z} / D}^{k+1}$ as in Construction 5.1. Then

$$
\begin{aligned}
\lim _{\substack{t \rightarrow 0 \\
t \neq 0}}\left\|\theta_{1}(t) \wedge \cdots \wedge \theta_{n}(t) \wedge \alpha_{1}(t) \wedge \cdots \wedge \beta_{b}^{(n)}(t)\right\| Q, t|t|^{n / 6}=\left\|\alpha_{1} \wedge \cdots \wedge \alpha_{a}\right\|_{Q} \\
\cdot\left\|\beta_{1}^{(1)} \wedge \cdots \wedge \beta_{b}^{(1)}\right\|_{Q} \cdot \cdots \cdot\left\|\beta_{1}^{(n)} \wedge \cdots \wedge \beta_{b}^{(n)}\right\|_{Q} .
\end{aligned}
$$

\subsection{Degeneracy of the Selberg zeta function in family}

Theorem 5.4 (Wolpert).

(i) Let $\gamma_{1}(t), \ldots, \gamma_{n}(t) \subset \mathfrak{Z}_{t}$ be the simple closed geodesics that are pinched to a node as $t \rightarrow 0$. Then the holomorphic function

$$
\frac{Z\left(\mathfrak{Z}_{t}, s\right)}{\prod_{j} Z_{l\left(\gamma_{j}(t)\right)}(s)}, \quad \operatorname{Re} s>\frac{1}{2},
$$

locally uniformly converges to $Z\left(X^{\circ}, s\right) \prod_{j} Z\left(T_{j}^{\circ}, s\right)$ as $t \rightarrow 0$.

(ii) The length $l\left(\gamma_{j}(t)\right)$ of the geodesic $\gamma_{j}(t)$ satisfies the estimate

$$
l\left(\gamma_{j}(t)\right)=\frac{2 \pi^{2}}{\log |t|^{-1}}+\mathcal{O}\left(\frac{1}{(\log |t|)^{4}}\right) \quad \text { as } t \rightarrow 0 .
$$

Proof. The first item is contained in [29, Ths. 35 and 38], [35, Proof of Conjs. 1 and 2]. The second item is [36, Ex. 4.3, p. 446].

Corollary 5.5. Let $k \geq 1$ be an integer. Then the Selberg zeta functions of the fibers $\mathfrak{Z}_{t}$ of the family $\mathfrak{g}: \mathfrak{Z} \rightarrow D$ satisfy

$$
\begin{aligned}
\lim _{\substack{t \rightarrow 0 \\
t \neq 0}} Z\left(\mathfrak{Z}_{t}, k+1\right)|t|^{-n / 6}\left(\log |t|^{-1}\right)^{-(2 k+1) n}= & \frac{1}{\left(\pi\left(2 \pi^{2}\right)^{2 k}(k !)^{2}\right)^{n}} \\
& \cdot Z\left(X^{\circ}, k+1\right) \prod_{j=1}^{n} Z\left(T_{j}^{\circ}, k+1\right) .
\end{aligned}
$$

Proof. First of all, from [29, Lemma 39], for $\operatorname{Re} s>0$ we have

$$
\lim _{l \rightarrow 0} \Gamma(s)^{2} Z_{l}(s) \exp \left(\pi^{2} / 3 l\right) l^{2 s-1}=2 \pi .
$$

We evaluate at $s=k+1$ and take Theorem 5.4(ii) into account. We find

$$
Z_{l\left(\gamma_{j}(t)\right)}(k+1) \sim \frac{1}{\pi\left(2 \pi^{2}\right)^{2 k}(k !)^{2}}|t|^{1 / 6}\left(\log |t|^{-1}\right)^{2 k+1} \quad \text { as } t \rightarrow 0 .
$$

The corollary is now a straightforward consequence of Theorem 5.4(i) and (5.1). 
5.2. Degeneracy of the family $L^{2}$ metric

Theorem 5.6. Let $k \geq 1$. For the family $L^{2}$ metric on $\operatorname{det} R \pi_{*} \omega_{\mathfrak{Z} / D}^{k+1} \mid D \backslash\{0\}$ we have

$$
\begin{aligned}
\lim _{\substack{t \rightarrow 0 \\
t \neq 0}} \| \theta_{1}(t) \wedge \cdots \wedge \theta_{n}(t) \wedge & \alpha_{1}(t) \wedge \cdots \wedge \beta_{b}^{(n)}(t) \|_{L^{2}, t}^{2}\left(\log |t|^{-1}\right)^{-n(2 k+1)} \\
= & \left(\frac{(2 k) !}{2^{k-1} \pi^{2 k}(k !)^{2}}\right)^{n}\left\|\alpha_{1} \wedge \cdots \wedge \alpha_{a}\right\|_{L^{2}}^{2} \\
& \cdot\left\|\beta_{1}^{(1)} \wedge \cdots \wedge \beta_{b}^{(1)}\right\|_{L^{2}}^{2} \cdot \cdots \cdot\left\|\beta_{1}^{(n)} \wedge \cdots \wedge \beta_{b}^{(n)}\right\|_{L^{2}}^{2} .
\end{aligned}
$$

The proof of the theorem is an adaptation of [27, Proof of Th. 2]. The arguments are detailed in the rest of the section.

Let $0<c<1$ be a small real constant and $t \in \mathbb{C}$ with $|t|<c$. Define $A_{t}=\{u \in \mathbb{C} \mid$ $|t| / c<|u|<c\}$. We denote by $\langle\cdot, \cdot\rangle_{t}$ the hermitian metric on the holomorphic tangent bundle $T_{A_{t}}$ characterized by

$$
\begin{aligned}
\left\langle\frac{\partial}{\partial u}, \frac{\partial}{\partial u}\right\rangle_{t} & =\frac{1}{2}\left(\frac{|u| \log |t|}{\pi} \sin \left(\frac{\pi \log |u|}{\log |t|}\right)\right)^{-2} \quad \text { if } t \neq 0, \\
\left\langle\frac{\partial}{\partial u}, \frac{\partial}{\partial u}\right\rangle_{0} & =\frac{1}{2} \frac{1}{|u|^{2}(\log |u|)^{2}} \quad \text { if } t=0 .
\end{aligned}
$$

We still write $\langle\cdot, \cdot\rangle_{t}$ for the metric induced on the tensor powers $T_{A_{t}}^{\otimes k}, k \in \mathbb{Z}$. The normalized Kähler form attached to $\langle\cdot, \cdot\rangle_{t}$ is

$$
\begin{aligned}
\omega_{t} & =\frac{i}{2 \pi}\left\langle\frac{\partial}{\partial u}, \frac{\partial}{\partial u}\right\rangle_{t} d u \wedge d \bar{u} \\
& =\frac{i}{4 \pi}\left(\frac{|u| \log |t|}{\pi} \sin \left(\frac{\pi \log |u|}{\log |t|}\right)\right)^{-2} d u \wedge d \bar{u} \quad \text { if } t \neq 0, \\
\omega_{0} & =\frac{i}{4 \pi} \frac{d u \wedge d \bar{u}}{|u|^{2}(\log |u|)^{2}} \quad \text { if } t=0 .
\end{aligned}
$$

Lemma 5.7. (i) For $0<|t|<c$,

$$
I_{1}(t):=\int_{A_{t}}\left\langle(d u / u)^{k+1},(d u / u)^{k+1}\right\rangle_{t} \omega_{t}=\frac{1}{2^{k-1} \pi^{2 k}}\left(\log |t|^{-1}\right)^{2 k+1} \frac{(2 k) !}{(k !)^{2}} R(t),
$$

where $R(t) \rightarrow 1$ as $t \rightarrow 0$.

(ii) Ast $\rightarrow 0$ we have

$$
I_{2}(t):=\int_{A_{t}}\left\langle u(d u / u)^{k+1},(d u / u)^{k+1}\right\rangle_{t} \omega_{t}=O(1) .
$$

Proof. The changes $u=\rho e^{i \theta}$ and $x=(\log \rho) / \log |t|$ reduce the first integral to

$$
I_{1}(t)=\frac{2^{k+1}}{\pi^{2 k}}\left(\log |t|^{-1}\right)^{2 k+1} \int_{\varepsilon}^{1-\varepsilon}(\sin (\pi x))^{2 k} d x,
$$


where $\varepsilon=(\log c) / \log |t|$. We conclude thanks to the identitiy $\int_{0}^{1}(\sin (\pi x))^{2 k} d x=$ $(2 k) ! /\left(2^{2 k}(k !)^{2}\right)$. The second integral is treated analogously.

Proposition 5.8. Let $0<|t|<c$. For the $L^{2}$-pairing $\langle\cdot, \cdot\rangle_{L^{2}, t}$ on $H^{0}\left(\mathfrak{Z}_{t}, \omega_{\mathfrak{Z}_{t}}^{k+1}\right)$, we have as $t \rightarrow 0$ :

(i) $\left\langle\theta_{i}(t), \theta_{j}(t)\right\rangle_{L^{2}, t}=O(1)$ if $i \neq j$;

(ii) $\left\langle\theta_{i}(t), \alpha_{j}(t)\right\rangle_{L^{2}, t}=O(1)$ for all $i, j$;

(iii) $\left\langle\theta_{i}(t), \beta_{l}^{(j)}(t)\right\rangle_{L^{2}, t}=O(1)$ for all $i, j, l$;

(iv) $\left\langle\theta_{i}(t), \theta_{i}(t)\right\rangle_{L^{2}, t}=\frac{1}{2^{k-1} \pi^{2 k}}\left(\log |t|^{-1}\right)^{2 k+1} \frac{(2 k) !}{(k !)^{2}} R(t)\left(1+O\left((\log |t|)^{-2}\right)\right)$ for all $i$.

Proof. Corresponding to every node of $\mathfrak{Z}_{0}$, there is a holomorphic embedding of the collar $C_{t}:=\left\{(u, v) \in \mathbb{C}^{2}|| t|/ c \leq| u|| v \mid, \leq c, u v=t\right\}$ into $\mathfrak{Z}_{t}$, provided $0<c<1$ is small enough. Observe that $A_{t}$ is then identified to the interior of $C_{t}$. This is presented in [12, Cons. 4.1]. By [36, Exp. 4.2], we are reduced to considering the integrals

$$
\int_{A_{t}}\left\langle\vartheta_{1}(t), \vartheta_{2}(t)\right\rangle_{t} \omega_{t}
$$

where $\left(\vartheta_{1}, \vartheta_{2}\right)$ is any choice of pairs of forms as in the statement and $\langle\cdot, \cdot\rangle_{t}, \omega_{t}$ are the metric and Kähler form defined by (5.2)-(5.3). We treat the case $\vartheta_{1}=\vartheta_{2}=\theta_{i}(t)$. The remaining cases are dealt with in a similar way. On $A_{t}$ we can decompose $\theta_{i}(t)=$ $(d u / u)^{k+1}\left(\theta_{i u}+\varepsilon+\theta_{i v}\right)$. Here $\varepsilon= \pm 1$ and the function $\theta_{i u}$ (resp. $\left.\theta_{i v}\right)$ is holomorphic on $|u|<c$ (resp. $|v|<c$ ) and vanishes at $u=0$ (resp. $v=0$ ). Following [27, Proof of Theorem 2, The first term], there are uniform bounds $\left|\theta_{i u}\right| \leq c^{\prime}|u|,\left|\theta_{i v}\right| \leq c^{\prime}|v|$, for some constant $c^{\prime}$ depending only on $\theta_{i}$. The claim then follows from Lemma 5.7(i).

Proposition 5.9. The functions

$$
\left\langle\alpha_{i}(t), \alpha_{j}(t)\right\rangle_{L^{2}, t}, \quad\left\langle\alpha_{i}(t), \beta_{l}^{(j)}(t)\right\rangle_{L^{2}, t}, \quad\left\langle\beta_{l}^{(j)}(t), \beta_{l^{\prime}}^{\left(j^{\prime}\right)}(t)\right\rangle_{L^{2}, t}
$$

continuously depend on $t \in D$.

Proof. Fix $\vartheta_{1}(t), \vartheta_{2}(t) \in\left\{\alpha_{i}(t), \beta_{l}^{(j)}(t)\right\}_{i, j, l}$. As in the proof of Proposition 5.8 we reduce to studying the continuity at 0 of the function

$$
F(t):=\int_{A_{t}}\left\langle\vartheta_{1}(t), \vartheta_{2}(t)\right\rangle_{t} \omega_{t}
$$

Here $A_{t}$ is the interior of a collar $C_{t}$ of a fixed developing node. On $A_{t}$ we decompose $\vartheta_{i}=(d u / u)^{k+1}\left(\vartheta_{i u}+\vartheta_{i v}\right), i=1,2$, where the functions $\vartheta_{i u}$ (resp. $\vartheta_{i v}$ ) are holomorphic in the domain $|u|<c$ (resp. $|v|<c$ ) and vanish at $u=0$ (resp. $v=0$ ). Hence there are uniform bounds $\left|\vartheta_{i u}\right| \leq c^{\prime}|u|$ and $\left|\vartheta_{i v}\right| \leq c^{\prime}|v|$, where $c^{\prime}$ only depends on $\vartheta_{1}, \vartheta_{2}$. Jointly with Lemma 5.7(i), this yields

$$
\left|\int_{A_{t}}\left(\vartheta_{1 u} \overline{\vartheta_{2 v}}+\vartheta_{1 v} \overline{\vartheta_{2 u}}\right)\left\langle(d u / u)^{k+1},(d u / u)^{k+1}\right\rangle_{t} \omega_{t}\right|=O\left(|t|\left(\log |t|^{-1}\right)^{2 k+1}\right) .
$$

The constant involved in the $O$ term depends only on $\vartheta_{1}$ and $\vartheta_{2}$. The quantity (5.4) tends to 0 as $t \rightarrow 0$. It remains to describe the contribution of the terms $\vartheta_{1 u} \overline{\vartheta_{2 u}}$ and $\vartheta_{1 v} \overline{\vartheta_{2 v}}$. 
We now focus on the term corresponding to $\vartheta_{1 u} \overline{\vartheta_{2 u}}$. The study of $\vartheta_{1 v} \overline{\vartheta_{2 v}}$ is performed analogously. The forms $\vartheta_{1 u}, \vartheta_{2 u}$ depend holomorphically on $t$ and vanish at $u=0$. According to [27, Proof of Theorem 2, The second term], the Schwarz lemma shows that $\vartheta_{1 u}(t)-\vartheta_{1 u}(0)$ and $\vartheta_{2 u}(t)-\vartheta_{2 u}(0)$ are bounded by $c^{\prime}|u t| \leq c^{\prime \prime}$. The constants $c^{\prime}, c^{\prime \prime}$ depend only on $\vartheta_{1}$ and $\vartheta_{2}$. The following expansion is then straightforward:

$$
\begin{aligned}
\int_{A_{t}} \vartheta_{1 u} \overline{\vartheta_{2 u}}\left\langle(d u / u)^{k+1}\right. & \left.,(d u / u)^{k+1}\right\rangle_{t} \omega_{t} \\
= & \int_{A_{t}} \vartheta_{1 u}(0) \overline{\vartheta_{2 u}}(0)\left\langle(d u / u)^{k+1},(d u / u)^{k+1}\right\rangle_{t} \omega_{t} \\
& +O\left(|t|(\log |t|)^{2 k}\right) .
\end{aligned}
$$

The constant involved in the $O$ term depends only on $\vartheta_{1}, \vartheta_{2}$. The quantity (5.7) converges to 0 as $t \rightarrow 0$. To achieve the conclusion, we are thus reduced to establishing the continuity of the function $G(t):=(5.6)$.

Split the domain $A_{t}=\{|t| / c<|u|<c\}$ into the union of $A_{1 t}:=\left\{|t|^{1 / 2} \leq|u|<c\right\}$ and $A_{2 t}:=\left\{|t| / c<|u| \leq|t|^{1 / 2}\right\}$. Accordingly, decompose $G(t)=G_{1}(t)+G_{2}(t)$. For the first, the function $x=\pi \log |u| / \log |t|$ on $A_{1 t}$ satisfies $0 \leq x \leq \pi / 2$. As in [27], we have $0 \leq x-\frac{1}{6} x^{3} \leq \sin x \leq x$ and $0 \leq x^{2}-(\sin x)^{2} \leq \frac{1}{4} x^{4}-\frac{1}{36} x^{6}$. Therefore

$$
x^{2 k}-(\sin x)^{2 k}=O\left(x^{2 k+2}\right), \quad x=\frac{\pi \log |u|}{\log |t|}, \quad u \in A_{1 t} .
$$

After an elementary algebraic manipulation, the estimate (5.8) yields

$$
\begin{aligned}
\left\langle(d u)^{k+1},(d u)^{k+1}\right\rangle_{t} \omega_{t} & =\left\langle(d u)^{k+1},(d u)^{k+1}\right\rangle_{0} \omega_{0} \\
& +O\left((\log |t|)^{-2}|u|^{2 k}(\log |u|)^{2 k+2} i d u \wedge d \bar{u}\right), \quad u \in A_{1 t} .
\end{aligned}
$$

Moreover, observe that $\left|\vartheta_{1 u}(0) \overline{\vartheta_{2 u}}(0)\right|=\mathcal{O}\left(|u|^{2}\right)$. Indeed, Schwarz' lemma ensures uniform bounds $\left|\vartheta_{1 u}\right|,\left|\vartheta_{2 u}\right| \leq c^{\prime}|u|$. Take this fact into account together with (5.9). After integration we get

$$
G_{1}(t)=\int_{A_{1 t}} \vartheta_{1 u}(0) \overline{\vartheta_{2 u}(0)}\left\langle(d u / u)^{k+1},(d u / u)^{k+1}\right\rangle_{0} \omega_{0}+O\left((\log |t|)^{-2}\right) .
$$

The $O$ term converges to 0 as $t \rightarrow 0$, whereas the integral on the right hand side of (5.10) is continuous - as a function of $t$-at $t=0$. For the second function $G_{2}(t)$, there is a trivial estimate

$$
\vartheta_{1 u} \overline{\vartheta_{2 u}}\left\langle(d u / u)^{k+1},(d u / u)^{k+1}\right\rangle_{t} \omega_{t}=O\left((\log |t|)^{2 k} i d u \wedge d \bar{u}\right), \quad u \in A_{2 t} .
$$

After integration over $A_{2 t}$, we find

$$
G_{2}(t)=O\left(|t|(\log |t|)^{2 k}\right),
$$

so $G_{2}(t) \rightarrow 0$ as $t \rightarrow 0$. The proposition results from (5.4)-(5.7) and (5.10)-(5.11). 
Proof of Theorem 5.6. For every $t \in D, t \neq 0$, introduce the matrix $H_{t}$ of the hermitian product $\langle\cdot, \cdot\rangle_{L^{2}, t}$ on $H^{0}\left(\mathfrak{Z}_{t}, \omega_{\mathfrak{Z}_{t}}\right)$ in the base $\theta_{i}(t), \alpha_{j}(t), \beta_{l}^{(k)}(t)$ :

$$
H_{t}=\left(\begin{array}{cc}
\left(\left\langle\theta_{i}(t), \theta_{j}(t)\right\rangle_{t}\right)_{i j} & \cdots \\
\vdots & B_{t}
\end{array}\right),
$$

where $B_{t}$ denotes the matrix

$$
\left(\begin{array}{cccc}
\left(\left\langle\alpha_{i}(t), \alpha_{j}(t)\right\rangle_{t}\right)_{i j} & \cdots & \cdots & \cdots \\
\vdots & \left(\left\langle\beta_{i}^{(1)}(t), \beta_{j}^{(1)}(t)\right\rangle_{t}\right)_{i, j} & \cdots & \vdots \\
\vdots & \vdots & \ddots & \vdots \\
\vdots & \cdots & \cdots & \left(\left\langle\beta_{i}^{(n)}(t), \beta_{j}^{(n)}(t)\right\rangle_{t}\right)_{i j}
\end{array}\right) .
$$

The conclusions of Propositions 5.8 and 5.9 guarantee that [27, Lemma 4] applies to $H_{t}$ :

$$
\operatorname{det} H_{t}=\operatorname{det} B_{t}\left(\prod_{i=1}^{n}\left\langle\theta_{i}(t), \theta_{i}(t)\right\rangle_{t}\right)(1+O(\delta)),
$$

with $\delta:=\sum_{i=1}^{n}\left\langle\theta_{i}(t), \theta_{i}(t)\right\rangle_{t}^{-1}$. The constant involved in the $O$ term does not depend on $t$. We insert the estimate of Proposition 5.8(iv) into equation (5.12). By the continuity property claimed by Proposition 5.9, we find

$$
\lim _{\substack{t \rightarrow 0 \\ t \neq 0}} \operatorname{det} H_{t}\left(\log |t|^{-1}\right)^{-n(2 k+1)}=\left(\frac{(2 k) !}{2^{k-1} \pi^{2 k}(k !)^{2}}\right)^{n} \operatorname{det} B_{0} .
$$

The proof of the theorem is now complete.

We are ready to finish the proof of Theorem 5.3.

Proof of Theorem 5.3. The theorem is an immediate application of Corollary 5.5, Theorem 5.6 and the definition of the Quillen metric (Definition 4.2).

\section{Metrized Mumford isomorphisms on $\mathcal{M}_{g, n}$}

This section focuses on an arakelovian counterpart of Theorem 3.5. We derive some consequences, such as the Takhtajan-Zograf local index theorem [33]-[34] and Theorems A and $\mathrm{B}$.

Recall from Section 4 the construction of the hermitian tautological line bundles $\lambda_{k+1 ; g, n ; Q}, \psi_{g, n ; W}, \delta_{g, n ; T}$ and $\kappa_{g, n ; F T}$. For the first three of them, the underlying metric is only defined on $\mathcal{M}_{g, n}^{\text {an }}$, while for the last one the metric is continuous on $\overline{\mathcal{M}}_{g, n}^{\text {an }}$ (Theorem 4.3).

Theorem 6.1 (Metrized Mumford isomorphism). Let $k \geq 0$ be an integer and $\mathcal{D}_{k+1 ; g, n}^{\circ}$ the restriction of the Mumford isomorphism $\mathcal{D}_{k+1 ; g, n}$ to $\mathcal{M}_{g, n}$. Then $\mathcal{D}_{k+1 ; g, n}^{\circ}$ induces an isometry

$$
\overline{\mathcal{D}}_{k+1 ; g, n}^{\circ}: \lambda_{k+1 ; g, n ; Q}^{\otimes 12} \otimes \psi_{g, n ; W} \stackrel{\sim}{\rightarrow} \kappa_{g, n ; F T}^{\otimes\left(6 k^{2}+6 k+1\right)} \otimes \mathcal{O}(C(g, n)) .
$$


The proof of the theorem follows the same pattern as for [12, Th. 6.1]. The first step consists in a simple remark: the case $g \geq 2, n=0$ is already known from the work of Deligne [6] and Gillet-Soulé [15].

Proposition 6.2. Let $k \geq 1, g \geq 2$ be integers.

(i) The conclusion of Theorem 6.1 holds true for $(g, n)=(g, 0)$.

(ii) The hermitian line bundle $\lambda_{k+1 ; g, 0 ; Q}^{\otimes 12} \otimes \delta_{g, n ; T}^{-1}$ extends to a continuous hermitian line bundle on $\overline{\mathcal{M}}_{g, 0}$. Moreover, $\overline{\mathcal{D}}_{k+1 ; g, 0}^{\circ}$ extends to an isometry of continuous hermitian line bundles on $\overline{\mathcal{M}}_{g, n}$

$$
\lambda_{k+1 ; g, 0 ; Q}^{\otimes 12} \otimes \delta_{g, 0 ; T}^{-1} \stackrel{\sim}{\rightarrow} \kappa_{g, 0 ; F T}^{\otimes\left(6 k^{2}+6 k+1\right)} \otimes \mathcal{O}(C(g, 0)) .
$$

Proof. The first assertion is due to Deligne [6, Th. 11.4] and Gillet-Soule [15]: it suffices to check that our definition of Quillen metric agrees with theirs. Let $X$ be a compact Riemann surface of genus $g \geq 2$. Endow the holomorphic tangent bundle $T_{X}$ with the hermitian metric attached to the riemannian hyperbolic metric of constant curvature -1 on $X$. The canonical sheaf $\omega_{X}$ is equipped with the dual metric. With these choices, our $L^{2}$ metric on $H^{0}\left(X, \omega_{X}^{k+1}\right)$ coincides with the one of Deligne and Gillet-Soulé. We still need to see that our normalization of the $L^{2}$ metric defining the Quillen metric (Definition 4.2) is given by $\exp \left(\frac{1}{2} T\left(\bar{\omega}_{X}^{k+1}\right)\right)$. Here $T\left(\bar{\omega}_{X}^{k+1}\right)$ is the analytic torsion of $\bar{\omega}_{X}^{k+1}$, for our choice of Kähler metric on $T_{X}$ (Section 4). The evaluation of $T\left(\bar{\omega}_{X}^{k+1}\right)$ has been indicated by Sarnak [28, p. 116, par. 1] and completed-for instance—in [8, Cor. 1.12]:

$$
T\left(\bar{\omega}_{X}^{k+1}\right)=-\log Z(X, k+1)-(2 g-2) C_{k},
$$

where $C_{k}$ is the constant defined in $\S 4.1$. Taking into account the definition of $E_{k+1}(g, 0)$, this proves the first claim. The second item already follows from (i), Theorem 3.5 and the continuity of the first tautological metric on $\kappa_{g, 0}$ on $\overline{\mathcal{M}}_{g, 0}$ (Theorem 4.3).

Proof of Theorem 6.1. The case $k=0$ is the content of [12, Th. 6.1]. For $k \geq 1$, due to Corollary 3.4, Corollary 3.7, Lemma 5.2, Theorem 5.3 and Proposition 6.2, the proof is formally the same as for $k=0$. We refer to [12, Sec. 6] for the details.

Corollary 6.3. Let $k \geq 1$ be an integer.

(i) The hermitian line bundle $\lambda_{k+1 ; g, n ; Q} \otimes \lambda_{k ; g, n ; Q}^{-1}$ on $\mathcal{M}_{g, n}$ extends to a continuous hermitian line bundle on $\overline{\mathcal{M}}_{g, n}$, whose underlying sheaf is $\lambda_{k+1 ; g, n} \otimes \lambda_{k ; g, n}^{-1}$.

(ii) There is an isometry of continuous hermitian line bundles on $\overline{\mathcal{M}}_{g, n}$

$$
\lambda_{k+1 ; g, n ; Q} \otimes \lambda_{k ; g, n ; Q}^{-1} \stackrel{\sim}{\rightarrow} \kappa_{g, n ; F T}^{\otimes k}
$$

Proof. First of all, from the isomorphism (3.8) of Lemma 3.6 we get an isomorphism

$$
\mathcal{D}: \lambda_{k+1 ; g, n} \otimes \lambda_{k ; g, n}^{-1} \stackrel{\sim}{\rightarrow} \kappa_{g, n}^{\otimes k}
$$


By [12, Cor. 3.2] and the Mumford isomorphisms (Theorem 3.5), $\mathcal{D}^{\otimes 12}$ coincides, up to a sign, with $\mathcal{D}_{k+1 ; g, n} \otimes \mathcal{D}_{k ; g, n}^{\otimes-1}$. Therefore, by the metrized Mumford isomorphisms (Theorem 6.1), $\left.\mathcal{D}\right|_{\mathcal{M}_{g, n}} ^{\otimes 12}$ induces an isometry

$$
\left.\overline{\mathcal{D}}\right|_{\mathcal{M}_{g, n}} ^{\otimes 12}:\left(\lambda_{k+1 ; g, n ; Q} \otimes \lambda_{k ; g, n ; Q}^{-1}\right)^{\otimes 12} \stackrel{\sim}{\rightarrow} \kappa_{g, n ; F T}^{\otimes 12 k}
$$

We conclude by (6.1)-(6.2) and the continuity of the first tautological metric on $\overline{\mathcal{M}}_{g, n}$ (Theorem 4.3).

Remark 6.4. Observe that the metric on $\lambda_{k+1 ; g, n ; Q} \otimes \lambda_{k ; g, n ; Q}^{-1}$, evaluated for a smooth $n$-pointed stable curve $\left(X ; p_{1}, \ldots, p_{n}\right)$ over $\mathbb{C}$, involves the factor $R^{\prime}(U, 1)$ if $k=0$ and $R(U, k+1)$ if $k \geq 1$, where $R(U, s)$ is the Ruelle zeta function of $U=X \backslash\left\{p_{1}, \ldots, p_{n}\right\} .^{3}$

Corollary 6.5 (Takhtajan-Zograf local index formula [33]-[34]). Let $\omega_{W P}, \omega_{T Z}$ be the Weil-Petersson and Takhtajan-Zograf Kähler forms on $\mathcal{M}_{g, n}^{\mathrm{an}}$, respectively. For every integer $k \geq 0$, the following equality of differential forms on $\mathcal{M}_{g, n}^{\text {an }}$ holds:

$$
\mathrm{c}_{1}\left(\lambda_{k+1 ; g, n ; Q}\right)=\frac{6 k^{2}+6 k+1}{12 \pi^{2}} \omega_{W P}-\frac{1}{9} \omega_{T Z}
$$

Proof. Firstly, there is an equality of differential forms on $\mathcal{M}_{g, n}^{\text {an }}$

$$
\mathrm{c}_{1}\left(\kappa_{g, n ; F T}\right)=\frac{1}{\pi^{2}} \omega_{W P} .
$$

For a proof we refer to [36] (case $n=0$ ) and [10, Ch. 5] (general case). Secondly, [37, Th. 5] proves the identity

$$
\mathrm{c}_{1}\left(\psi_{g, n ; W}\right)=\frac{4}{3} \omega_{T Z}
$$

The relation (6.3) is obtained from Theorem 6.1 and (6.4)-(6.5).

Proof of Theorem A. The theorem is a consequence of Theorems 3.5 and 6.1. The argument is the same as for [12, Th. A].

Proof of Theorem B. Let $\mathcal{U}=\mathcal{X} \backslash \bigcup_{j} \sigma_{j}(\mathcal{S})$. By Theorem A(ii), it suffices to show that for every complex embedding $\sigma: K \hookrightarrow \mathbb{C}$,

$$
\log \left(E_{k+1}(g, n) Z\left(\mathcal{U}_{\sigma}(\mathbb{C}), k+1\right)\right)=\mathcal{O}(k \log k) .
$$

On the one hand, the product representation of the Selberg zeta function (see Section 4.1) provides $\log Z\left(\mathcal{U}_{\sigma}(\mathbb{C}), k+1\right)=o(1)$. It remains to prove that $\log E_{k+1}(g, n)=$ $\mathcal{O}(k \log k)$. From the definition of $E_{k+1}(g, n)$, the relevant terms to treat are:

(i) terms in $\log (2 k)$ !; these $\operatorname{are} \mathcal{O}(k \log k)$ by Stirling's formula;

\footnotetext{
3 Recall that $R(U, s)=Z(U, s) / Z(U, s+1)$, for $Z$ the Selberg zeta function of $U$.
} 
(ii) $\alpha_{k}:=\left(\sum_{j=1}^{2 k}(j-k) \log j\right)-k^{2}$; we can write

$$
\alpha_{k}=\log \Gamma_{2}(2 k+1)+k \log (2 k) !-k^{2},
$$

where $\Gamma_{2}$ is the Barnes double gamma function [2] (see also [28]). By [2] and Stirling's formula we have

$$
\begin{aligned}
\log \Gamma_{2}(2 k+1) & =(2 k)^{2}\left(\frac{\log (2 k)}{2}-\frac{3}{4}\right)+\mathcal{O}(k), \\
k \log (2 k) ! & =2 k^{2} \log (2 k)-3 k^{2}+\mathcal{O}(k \log k) .
\end{aligned}
$$

From the expansions (6.6)-(6.7) we infer $\alpha_{k}=\mathcal{O}(k \log k)$.

The proof is complete.

\section{Applications to pointed stable curves of genus 0}

Let $n \geq 3$ be an integer. In this section we denote by $\overline{\mathbf{M}}_{0, n}$ the moduli space of isomorphism classes of pointed stable curves of genus 0 . It is a smooth and projective scheme over Spec $\mathbb{Z}$. We write $\mathbf{M}_{0, n}$ for the open subscheme classifying smooth curves. There is a natural projection $\overline{\mathcal{M}}_{0, n} \rightarrow \overline{\mathbf{M}}_{0, n}$. The contraction morphism $\overline{\mathcal{M}}_{0, n+1} \rightarrow \overline{\mathcal{M}}_{0, n}$ and the universal sections $\sigma_{1}, \ldots, \sigma_{n}$ descend to $\overline{\mathbf{M}}_{0, n+1} \rightarrow \overline{\mathbf{M}}_{0, n}$ with sections $\tau_{1}, \ldots, \tau_{n}$. The tuple $\left(\overline{\mathbf{M}}_{0, n+1} \rightarrow \overline{\mathbf{M}}_{0, n} ; \tau_{1}, \ldots, \tau_{n}\right)$ is the universal object for $n$-pointed stable curves of genus 0 , up to isomorphism. ${ }^{4}$ The line bundles $\lambda_{0, n ; k}, \psi_{0, n}, \delta_{0, n}$ and $\kappa_{0, n}$ descend to $\overline{\mathbf{M}}_{0, n}$, as also do the Quillen, Wolpert and first tautological metrics. We employ the same symbols for the descended objects. In the following we need the ampleness of $\kappa_{0, n}$ on $\overline{\mathbf{M}}_{0, n}$. For the sake of completeness, we prove this fact in the appendix.

\subsection{Heights of points with respect to $\psi_{0, n ; W}$}

Fix $n \geq 3$ an integer and $K$ a number field. Write $\mathcal{S}=\operatorname{Spec} \mathcal{O}_{K}$. Let $p: \operatorname{Spec} K \rightarrow \mathbf{M}_{0, n}$ be a $K$-valued point. Because $\overline{\mathbf{M}}_{0, n}$ is a projective scheme, there is a generically smooth pointed stable curve $\left(\pi: \mathcal{X} \rightarrow \mathcal{S} ; \wp_{1}, \ldots, \wp_{n}\right)$ with a cartesian diagram

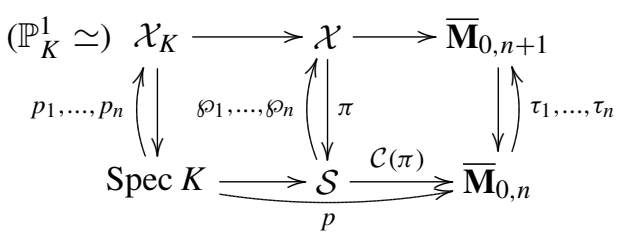

The real number $\widehat{\operatorname{deg}}\left(\mathcal{C}(\pi)^{*} \psi_{0, n ; W}\right)$ (resp. $\widehat{\operatorname{deg}}\left(\mathcal{C}(\pi)^{*} \bar{\kappa}_{0, n ; F T}\right)$ ) is well defined and only depends on the isomorphism class of $\left(\mathcal{X}_{K} ; p_{1}, \ldots, p_{n}\right)$. Indeed, $\mathcal{C}(\pi)$ is the unique extension of $p$ to a morphism $\operatorname{Spec} \mathcal{O}_{K} \rightarrow \overline{\mathbf{M}}_{0, n}$. For any object $Z$ in this isomorphism class, we write $h_{\psi}(Z)$ (resp. $\left.h_{\kappa}(Z)\right)$ for this quantity. Notice that $h_{\psi}$ (resp. $h_{\kappa}$ ) is defined for every smooth $n$-pointed stable curve of genus 0 .

\footnotetext{
4 The construction of [20] easily carries over to Spec $\mathbb{Z}$. This is written in [10, Part II, Chap. 1.5].
} 
Theorem 7.1. Let $n \geq 3$ be an integer and $K$ a fixed number field. Let $A$ be a real number. Up to isomorphism, there are finitely many smooth n-pointed stable curves $Z$ of genus 0 over $K$ with $h_{\psi}(Z) \leq A$.

Proof. We first claim the corresponding property is true for $h_{\kappa}$. Indeed, with the notation as in (7.1), if $Z \simeq\left(\mathcal{X}_{K} ; p_{1}, \ldots, p_{n}\right)$, then $h_{\kappa}(Z)$ is the height of the image of $\mathcal{C}(\pi)$ with respect to $\kappa_{0, n ; F T}$. Moreover $\kappa_{0, n}$ is ample on $\overline{\mathbf{M}}_{0, n}$ and the first tautological metric is continuous (Theorem 4.3). The assertion is a consequence of Northcott's finiteness property [30, Cor. 3.4]. Therefore, it is enough to show that a bound $h_{\psi}(Z) \leq A$ leads to a bound $h_{K}(Z) \leq B(n, K, A)$ for some real constant $B(n, K, A)$ depending only on $n, K$ and $A$. Observe that

$$
h_{\kappa}(Z)=\left(\omega \mathcal{X} / \mathcal{S}\left(\wp_{1}+\cdots+\wp_{n}\right)_{\text {hyp }}\right)^{2}
$$

for a model $\left(\pi: \mathcal{X} \rightarrow \mathcal{S} ; \wp_{1}, \ldots, \wp_{n}\right)$ of $Z$. We apply Theorem $\mathrm{A}\left(\right.$ ii), with $k=0 .^{5}$ Because we are in genus 0 , we have

$$
\begin{aligned}
\widehat{\operatorname{deg}} \widehat{c}_{1}\left(\lambda(\omega \mathcal{X} / \mathcal{S})_{Q}\right)= & \frac{1}{2} \sum_{\sigma: K_{\hookrightarrow} \mathbb{C}} \log \left(Z^{\prime}\left(\mathcal{U}_{\sigma}, 1\right)\right)+\frac{[K: \mathbb{Q}]}{2} \log E_{1}(0, n) \\
& -\frac{[K: \mathbb{Q}]}{2} \log (n-2),
\end{aligned}
$$

where $\mathcal{U}_{\sigma}=\mathcal{X}_{\sigma}(\mathbb{C}) \backslash\left\{p_{1}^{\sigma}, \ldots, p_{n}^{\sigma}\right\}$. Also,

$$
\widehat{\operatorname{deg}} \Delta \mathcal{X} / \mathcal{S} \geq 0
$$

Thus Theorem A(ii) provides an inequality

$$
h_{\psi}(Z)+6 \sum_{\sigma: K \hookrightarrow \mathbb{C}} \log Z^{\prime}\left(\mathcal{U}_{\sigma}, 1\right)+\alpha(n, K) \geq h_{\kappa}(Z)
$$

for some constant $\alpha(n, K)$ depending only on $n$ and $K$. We reduce to showing that there exists a constant $C$ such that for any $n$-punctured sphere $U=\mathbb{P}^{1}(\mathbb{C}) \backslash\left\{q_{1}, \ldots, q_{n}\right\}$, we have $Z^{\prime}(U, 1) \leq C$. Fix any 1 -pointed stable curves of genus $1,\left(T_{j}, t_{j}\right), j=1, \ldots, n$. We build the stable curve of genus $n$

$$
Y=\left(\mathbb{P}^{1}(\mathbb{C}) \sqcup T_{1} \sqcup \cdots \sqcup T_{n}\right) /\left(q_{1} \sim t_{1}, \ldots, q_{n} \sim t_{n}\right) .
$$

Let $\mathfrak{g}: \mathfrak{Z} \rightarrow D(0, \varepsilon)$ be a one-parameter small stable deformation of $Y$, whose only singular fiber is $\mathfrak{g}^{-1}(0)=\mathfrak{Z}_{0}=Y$. By [35, Conj. 1, p. 286], ${ }^{6}$, there exist constants $c, \beta>0$ depending only on $n$ such that

$$
\frac{Z^{\prime}\left(\mathfrak{Z}_{t}, 1\right)}{\prod_{\gamma, l(\gamma)<c} Z_{l(\gamma)}(1) \prod_{0<\lambda<1 / 4} \lambda} \leq \beta, \quad t \neq 0 .
$$

The product $\prod_{\gamma, l(\gamma)<c}$ is finite and runs over all closed primitive hyperbolic geodesics $\gamma$ of $\mathfrak{Z}_{t}$ of length $l(\gamma)<c$. Also, $Z_{l(\gamma)}(s)$ is the contribution of the geodesic $\gamma$ to the product

\footnotetext{
5 Strictly speaking [12, Th. A] does not apply, because it requires $\mathcal{X}$ to be regular.

6 In the cited statement, the expression $0<\lambda_{n}<1 / 4$ should actually read $0 \leq \lambda_{n}<1 / 4$.
} 
defining the Selberg zeta function $Z\left(\mathfrak{Z}_{t}, s\right)$ (see Section 4). Finally, the product $\prod_{0<\lambda<1 / 4}$ is finite and runs over eigenvalues of the hyperbolic laplacian on $\mathfrak{Z}_{t}$. Write $\gamma_{j}(t), j=$ $1, \ldots, n$, for the geodesics in $\mathfrak{Z}_{t}$ pinched to a node as $t \rightarrow 0$, and $\lambda_{1}(t), \ldots, \lambda_{n}(t)$ for the non-trivial eigenvalues converging to 0 [12, Th. 5.2]. We may assume $l\left(\gamma_{j}(t)\right)<c$ and $\lambda_{j}(t)<1 / 4$ for all $j, t \neq 0$. Observe that in (7.2) the rough inequalities $Z_{l(\gamma)}(1) \leq 1$, $\lambda \leq 1$ hold. It follows that

$$
\frac{Z^{\prime}\left(\mathfrak{Z}_{t}, 1\right)}{\prod_{j=1}^{n} Z_{l\left(\gamma_{j}(t)\right)}(1) \prod_{j=1}^{n} \lambda_{j}(t)} \leq \beta, \quad t \neq 0 .
$$

In [12, Proof of Th. 5.3] we show that (7.3) converges to

$$
Z^{\prime}(U, 1) \prod_{j=1}^{n} Z^{\prime}\left(T_{j} \backslash\left\{t_{j}\right\}, 1\right)
$$

as $t \rightarrow 0$. We infer that

$$
Z^{\prime}(U, 1) \leq \frac{\beta}{\prod_{j=1}^{n} Z^{\prime}\left(T_{j} \backslash\left\{t_{j}\right\}, 1\right)} .
$$

This concludes the proof.

\subsection{Integral cusp forms with small Petersson norm}

Let $K$ be a number field, with ring of integers denoted $\mathcal{O}_{K}$. Set $\mathcal{S}=\operatorname{Spec} \mathcal{O}_{K}$. Consider a pointed stable curve $\left(\pi: \mathcal{X} \rightarrow \mathcal{S} ; \sigma_{1}, \ldots, \sigma_{n}\right)$ and define $\mathcal{U}=\mathcal{X} \backslash \bigcup_{j} \sigma_{j}(\mathcal{S})$. Assume that $\pi$ is generically smooth. Recall the definition of the $L^{2}$ metric in Section 4. For every integer $k \geq 0$ and every field embedding $\sigma: K \hookrightarrow \mathbb{C}$, the complex vector space $H^{0}\left(\mathcal{X}, \omega_{\mathcal{X} / \mathcal{S}}^{k+1}\left(k \sigma_{1}+\cdots+k \sigma_{n}\right)\right) \otimes_{\sigma} \mathbb{C}$ comes equipped with the $L^{2}$ metric attached to the hyperbolic metric on $\mathcal{U}_{\sigma}(\mathbb{C})$; denote it by $\langle\cdot, \cdot\rangle_{L^{2}, \sigma}$. On the other hand, by the uniformization theorem, there is a biholomorphism $\mathcal{U}_{\sigma}(\mathbb{C}) \simeq \Gamma_{\sigma} \backslash \mathbb{H}$ for some discrete torsion subgroup $\Gamma_{\sigma} \subset \mathrm{PSL}_{2}(\mathbb{R})$. The space $S_{2 k+2}\left(\Gamma_{\sigma}\right)$ of modular cusp forms of weight $2 k+2$ for $\Gamma_{\sigma}$ is isomorphic to $H^{0}\left(\mathcal{X}, \omega_{\mathcal{X} / \mathcal{S}}^{k+1}\left(k \sigma_{1}+\cdots+\sigma_{n}\right)\right) \otimes_{\sigma} \mathbb{C}$. The Petersson metric on $S_{2 k+2}\left(\Gamma_{\sigma}\right)$ induces a hermitian metric on $H^{0}\left(\mathcal{X}, \omega_{\mathcal{X} / \mathcal{S}}^{k+1}\left(k \sigma_{1}+\cdots+k \sigma_{n}\right)\right) \otimes_{\sigma} \mathbb{C}$, to be denoted $\langle\cdot, \cdot\rangle_{\mathrm{Pet}, \sigma}$. It is readily checked that $\langle\cdot, \cdot\rangle_{L^{2}, \sigma}=\left(2^{k} / \pi\right)\langle\cdot, \cdot\rangle_{\mathrm{Pet}, \sigma}$. This relation stems from the comparison between the hermitian and riemannian hyperbolic metrics, as well as the normalization of the Kähler form $\omega$ (Section 4).

The space of global sections $H^{0}\left(\omega_{\mathcal{X} / \mathcal{S}}^{k+1}\left(k \sigma_{1}+\cdots+k \sigma_{n}\right)\right)$ is a projective $\mathcal{O}_{K}$-module. As a $\mathbb{Z}$-module, it becomes a lattice in the real vector space

$$
\begin{aligned}
H^{0}\left(\mathcal{X}, \omega_{\mathcal{X} / \mathcal{S}}^{k+1}\left(k \sigma_{1}+\cdots\right.\right. & \left.\left.+k \sigma_{n}\right)\right) \otimes_{\mathbb{Z}} \mathbb{C} \\
& =\left(\bigoplus_{\sigma: K \hookrightarrow \mathbb{C}} H^{0}\left(\mathcal{X}, \omega_{\mathcal{X} / \mathcal{S}}^{k+1}\left(k \sigma_{1}+\cdots+k \sigma_{n}\right)\right) \otimes_{\sigma} \mathbb{C}\right)^{F_{\infty}}
\end{aligned}
$$


This vector space is endowed with the norms

$$
\|f\|_{L^{2}, \infty}=\sup _{\sigma: K \hookrightarrow \mathbb{C}}\left\|f_{\sigma}\right\|_{L^{2}, \sigma} \quad \text { and } \quad\|f\|_{\text {Pet, } \infty}=\sup _{\sigma: K \hookrightarrow \mathbb{C}}\left\|f_{\sigma}\right\|_{\text {Pet, } \sigma} .
$$

Proposition 7.2. Assume $\left(\omega \mathcal{X} / \mathcal{S}\left(\sigma_{1}+\cdots+\sigma_{n}\right)_{\mathrm{hyp}}^{2}\right)>0$. Then there exists an integer $k_{0} \geq 0$ such that

$$
\left\{f \in H^{0}\left(\omega_{\mathcal{X} / \mathcal{S}}^{k+1}\left(k \sigma_{1}+\cdots+k \sigma_{n}\right)\right) \mid\|f\|_{\text {Pet }, \infty}^{2} \leq \pi / 2^{k}\right\} \neq \emptyset \quad \text { for all } k \geq k_{0} .
$$

Proof. By Theorem B and Minkowski's theorem, we infer that there exists $k_{0} \geq 0$ such that

$$
\left\{f \in H^{0}\left(\omega_{\mathcal{X} / \mathcal{S}}^{k+1}\left(k \sigma_{1}+\cdots+k \sigma_{n}\right)\right) \mid 0<\|f\|_{L^{2}, \infty} \leq 1\right\} \neq \emptyset \quad \text { for all } k \geq k_{0} .
$$

See [15, Sec. 5.2], [31, Ch. VIII, Par. 2.3] or [32, Exp. III, Th. 4] for the details of the argument. We conclude by the relation $\|f\|_{\mathrm{Pet}, \infty}^{2}=\left(\pi / 2^{k}\right)\|f\|_{L^{2}, \infty}^{2}$.

We keep the notation of $\S 7.1$. For a smooth $n$-pointed stable curve $Z$ of genus 0 over $K$ we write $\left(\pi: \mathcal{X} \rightarrow \mathcal{S} ; \wp_{1}, \ldots, \wp_{n}\right)$ for the model that fits into the cartesian diagram (7.1).

Theorem 7.3. Let $K$ be a fixed number field and $n \geq 3$ an integer. Up to isomorphism, for all but finitely many smooth n-pointed stable curves $Z$ of genus 0 over $K$, there exists an integer $k_{0}$ (depending on the isomorphism class of $Z$ ) such that

$$
\left\{f \in H^{0}\left(\mathcal{X}, \omega_{\mathcal{X} / \mathcal{S}}^{k+1}\left(k \wp_{1}+\cdots+k \wp_{n}\right)\right) \mid 0<\|f\|_{\text {Pet }, \infty}^{2} \leq \pi / 2^{k}\right\} \neq \emptyset \quad \text { for all } k \geq k_{0} .
$$

Proof. By Proposition 7.2, we have to show that there are finitely many isomorphism classes of such $Z$ with $h_{\kappa}(Z)=\left(\omega \mathcal{X} / \mathcal{S}\left(\wp_{1}+\cdots+\wp_{n}\right)_{\text {hyp }}^{2}\right) \leq 0$. Because $K$ has been fixed, $\kappa_{0, n ; F T}$ is ample and the first tautological metric is continuous, this follows from Northcott's finiteness property.

\section{Appendix}

Let $n \geq 3$ be an integer and $\overline{\mathbf{M}}_{0, n} \rightarrow$ Spec $\mathbb{Z}$ the moduli scheme of isomorphism classes of $n$-pointed stable curves of genus 0 . The contraction map $\overline{\mathbf{M}}_{0, n+1} \rightarrow \overline{\mathbf{M}}_{0, n}$ is endowed with sections $\tau_{1}, \ldots, \tau_{n}$. In this appendix we establish the ampleness of the line bundle $\kappa_{0, n}=\left\langle\omega_{\overline{\mathbf{M}}_{0, n+1} / \overline{\mathbf{M}}_{0, n}}\left(\tau_{1}+\cdots+\tau_{n}\right), \omega_{\overline{\mathbf{M}}_{0, n+1} / \overline{\mathbf{M}}_{0, n}}\left(\tau_{1}+\cdots+\tau_{n}\right)\right\rangle$.

Lemma 8.1 (Keel-Tevelev). Let $k$ be any field.

(i) The line bundle $\kappa_{0, n ; k}$ on $\overline{\mathbf{M}}_{0, n ; k}$ is very ample.

(ii) For every integer $m \geq 1$, let $W_{m}$ be the standard irreducible representation of the symmetric group $\mathfrak{S}_{m}$, i.e. $m$-tuples of integers that sum to 0 . Then

$$
H^{0}\left(\overline{\mathbf{M}}_{0, n ; k}, \kappa_{0, n ; k}\right) \simeq \bigotimes_{4 \leq j \leq n} W_{j}
$$

In particular $\operatorname{dim} H^{0}\left(\overline{\mathbf{M}}_{0, n ; k}, \kappa_{0, n ; k}\right)$ does not depend on the field $k$. 
Proof. This is Corollary 2.6 and Corollary 2.7 of [21] (observe that in Section 2 there, $k$ is an arbitrary field).

Theorem 8.2. The line bundle $\kappa_{0, n}$ on $\overline{\mathbf{M}}_{0, n}$ is ample.

Proof. We check that $\kappa_{0, n}$ satisfies the cohomological criterion of ampleness [18, Ch. III, Prop. 5.3]. Let $\mathcal{F}$ be a coherent sheaf on $\overline{\mathbf{M}}_{0, n}$. We must prove that there exists an integer $N_{0}>0$ such that

$$
H^{i}\left(\overline{\mathbf{M}}_{0, n}, \mathcal{F} \otimes \kappa_{0, n}^{\otimes N}\right)=0 \quad \text { for } N \geq N_{0} \text { and } i>0 .
$$

A first reduction consists in restricting to the class of coherent sheaves which are flat over Spec $\mathbb{Z}$. Indeed, suppose we have checked the criterion of ampleness for such sheaves. Since $\overline{\mathbf{M}}_{0, n}$ is projective over Spec $\mathbb{Z}$, for any coherent sheaf $\mathcal{F}$ on $\overline{\mathbf{M}}_{0, n}$ there is an exact sequence of coherent sheaves

$$
0 \rightarrow \mathcal{K} \rightarrow \mathcal{E} \rightarrow \mathcal{F} \rightarrow 0
$$

with $\mathcal{E}$ locally free. In particular, $\mathcal{E}$ is flat over $\overline{\mathbf{M}}_{0, n}$. Since $\overline{\mathbf{M}}_{0, n}$ is flat over Spec $\mathbb{Z}$, so is $\mathcal{E}$. Because $\mathbb{Z}$ is a principal ideal domain, flatness of $\mathcal{E}$ over Spec $\mathbb{Z}$ implies flatness of $\mathcal{K}$ over Spec $\mathbb{Z}$. Fix an integer $N_{0}$ such that (8.1) holds for both $\mathcal{K}$ and $\mathcal{E}$. Then (8.1) also holds for $\mathcal{F}$, by the long exact sequence of cohomology of (8.2). Henceforth $\mathcal{F}$ denotes a coherent sheaf flat over Spec $\mathbb{Z}$.

By Lemma 8.1 the line bundle $\kappa_{0, n ; \mathbb{Q}}$ is very ample on $\overline{\mathbf{M}}_{0, n ; \mathbb{Q}}$. If $\pi: \overline{\mathbf{M}}_{0, n} \rightarrow$ Spec $\mathbb{Z}$ stands for the structure map, then the natural morphism

$$
\pi^{*} \pi_{*} \kappa_{0, n} \rightarrow \kappa_{0, n}
$$

becomes surjective when restricted to $\overline{\mathbf{M}}_{0, n ; \mathbb{Q}}$, by global generation of $\kappa_{0, n} ; \mathbb{Q}$. Therefore, for a sufficiently divisible integer $l,(8.3)$ becomes surjective when restricted to $\overline{\mathbf{M}}_{0, n ; \mathbb{Z}[1 / l]}$. This yields a commutative diagram of proper morphisms

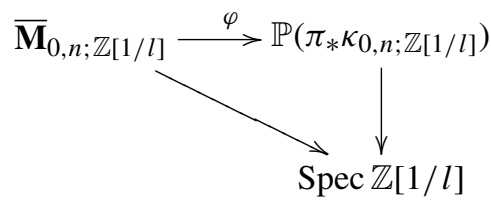

From Lemma 8.1 the dimension of $H^{0}\left(\overline{\mathbf{M}}_{0, n ; \mathbb{F}_{p}}, \kappa_{0, n ; \mathbb{F}_{p}}\right)$ is independent of $p$. By Grauert's theorem [18, Ch. III, Cor. 12.9], the sheaf $\pi_{*} \kappa_{0, n ; \mathbb{Z}[1 / l]}$ is locally free on $\operatorname{Spec} \mathbb{Z}[1 / l]$. In addition, for every $s \in \operatorname{Spec} \mathbb{Z}[1 / l]$ we have an isomorphism

$$
\pi_{*} \kappa_{0, n ; \mathbb{Z}[1 / l]} \otimes k(s) \stackrel{\sim}{\rightarrow} H^{0}\left(\overline{\mathbf{M}}_{0, n ; k(s)}, \kappa_{0, n ; k(s)}\right) .
$$

If $p$ is a prime number with $p \nmid l$, the isomorphism (8.5) amounts to

$$
\pi_{*} \kappa_{0, n ; \mathbb{Z}[1 / l]} \otimes_{\mathbb{Z}} \mathbb{F}_{p} \stackrel{\sim}{\rightarrow} H^{0}\left(\overline{\mathbf{M}}_{0, n ; \mathbb{F}_{p}}, \kappa_{0, n ; \mathbb{F}_{p}}\right)
$$


Therefore, reducing (8.4) modulo $p$ with $p \nmid l$ yields

$$
\overline{\mathbf{M}}_{0, n ; \mathbb{F}_{p}} \stackrel{\varphi_{p}}{\rightarrow} \mathbb{P}\left(H^{0}\left(\overline{\mathbf{M}}_{0, n ; \mathbb{F}_{p}}, \kappa_{0, n ; \mathbb{F}_{p}}\right)\right) .
$$

The morphism $\varphi_{p}$ is a closed immersion, since $\kappa_{0, n ; \mathbb{F}_{p}}$ is very ample. This being true for all $p \nmid l$, the morphism $\varphi$ of (8.4) is also a closed immersion. We infer that $\kappa_{0, n ; \mathbb{Z}[1 / l]}$ is very ample. Hence, there exists an integer $N_{0}>0$ such that

$$
H^{i}\left(\overline{\mathbf{M}}_{0, n ; \mathbb{Z}[1 / l]}, \mathcal{F} \otimes \kappa_{0, n ; \mathbb{Z}[1 / l]}^{\otimes N}\right)=0 \quad \text { for } N \geq N_{0} \text { and } i>0 .
$$

Since higher direct images commute with flat base change [18, Ch. III, Prop. 9.3], (8.6) equivalently reads

$$
R^{i} \pi_{*}\left(\mathcal{F} \otimes \kappa_{0, n}^{\otimes N}\right)(\operatorname{Spec} \mathbb{Z}[1 / l])=0 \quad \text { for } N \geq N_{0} \text { and } i>0 .
$$

We still need to deal with primes $p \mid l$. Let $p$ be such a prime. Since $\kappa_{0, n ; \mathbb{F}_{p}}$ is very ample, there exists $N_{p}>0$ for which the ampleness criterion holds for $\kappa_{0, n ; \mathbb{F}_{p}}$ and $\mathcal{F}_{\mathbb{F}_{p}}$. Since there are only finitely many primes dividing $l$, after possibly increasing $N_{0}$ we can suppose that $N_{0} \geq N_{p}$ for all $p \mid l$. Let us fix an integer $N \geq N_{0}$. For every $p \mid l$ and $i=1, \ldots, n-2$, introduce

$$
\Omega_{p, i}=\left\{y \in \operatorname{Spec} \mathbb{Z} \mid H^{i}\left(\overline{\mathbf{M}}_{0, n ; k(y)}, \mathcal{F} \otimes \kappa_{0, n ; k(y)}^{\otimes N}\right)=0\right\} .
$$

Then $\Omega_{p, i}$ is Zariski open by the semi-continuity theorem [18, Ch. III, Th. 12.8], and contains $p$ because $N \geq N_{0} \geq N_{p}$. We write

$$
\Omega_{p}=\bigcap_{i=0}^{n-2} \Omega_{p, i}
$$

which is a Zariski open neighborhood of $p$. Let $l_{p} \geq 1$ be an integer such that $p \in$ Spec $\mathbb{Z}\left[1 / l_{p}\right] \subseteq \Omega_{p}$. The construction of $\Omega_{p}$ and the semi-continuity theorem ensure

$$
R^{i} \pi_{*}\left(\mathcal{F} \otimes \kappa_{0, n}^{\otimes N}\right)\left(\operatorname{Spec} \mathbb{Z}\left[1 / l_{p}\right]\right)=0 \quad \text { for } i=1, \ldots, n-2 .
$$

Finally, observe that $\mathcal{U}:=\{\operatorname{Spec} \mathbb{Z}[1 / l]\} \cup\left\{\operatorname{Spec} \mathbb{Z}\left[1 / l_{p}\right]\right\}_{p \mid l}$ is an affine open covering of $\operatorname{Spec} \mathbb{Z}$. From (8.7)-(8.8),

$$
H^{i}\left(\overline{\mathbf{M}}_{0, n}, \mathcal{F} \otimes \kappa_{0, n}^{\otimes N}\right)=R^{i} \pi_{*}\left(\mathcal{F} \otimes \kappa_{0, n}^{\otimes N}\right)(\operatorname{Spec} \mathbb{Z})=0 \quad \text { for } i=1, \ldots, n-1 .
$$

This even holds for $i>n-2$, because $\overline{\mathbf{M}}_{0, n}$ has Krull dimension $n-2$. The proof is complete.

Acknowledgments. It is a pleasure to thank J.-B. Bost and J. I. Burgos, who proposed me to study the topic of this article during my $\mathrm{PhD}$. thesis, as well as a possible approach: it revealed to be fruitful. S. Wolpert kindly guided me at some points and shared his knowledge in Teichmüller theory. He also communicated to me the reference [27], crucial to this work. I am deeply indebted to him. I acknowledge stimulating discussions with J.-M. Bismut on questions related to this paper. Finally I am grateful to T. Hahn, J. Kramer, U. Kühn for the mathematical exchanges we had in the last years. 


\section{References}

[1] Artin, M., Grothendieck, A., Verdier, J.-L.: Séminaire de Géométrie Algébrique du BoisMarie 1963/64, tome 3, Lecture Notes in Math. 305, Springer (1973) Zbl 0245.00002

[2] Barnes, E. W.: The theory of the $G$-function. Quart. J. Math. 31, 264-314 (1900) JFM 30.0389.02

[3] Bost, J.-B.: Potential theory and Lefschetz theorems for arithmetic surfaces. Ann. Sci. École Norm. Sup. (4) 32, 241-312 (1999) Zbl 0931.14014 MR 1681810

[4] Burgos Gil, J. I., Kramer, J., Kühn, U.: Cohomological arithmetic Chow rings. J. Inst. Math. Jussieu 6, 1-172 (2007) Zbl 1115.14013 MR 2285241

[5] Burgos Gil, J. I., Kramer, J., Kühn, U.: Arithmetic characteristic classes of automorphic vector bundles. Documenta Math. 10, 619-716 (2005) Zbl 1080.14028 MR 2218402

[6] Deligne, P.: Le déterminant de la cohomologie. In: Current Trends in Arithmetical Algebraic Geometry (Arcata, CA, 1985), Contemp. Math. 67, Amer. Math. Soc., 93-177 (1987) Zbl 0629.14008 MR 0902592

[7] Deligne, P., Mumford, D.: The irreducibility of the space of curves of given genus. Publ. Math. IHES 36, 75-109 (1969) Zbl 0181.48803 MR 0262240

[8] Ebel, T.: Equivariant analytic torsion on hyperbolic Riemann surfaces and the arithmetic Lefschetz trace of an Atkin-Lehner involution on a compact Shimura curve. Doctoral Thesis, Heinrich-Heine Universität (2006)

[9] Elkik, R.: Fibrés d'intersection et intégrales de classes de Chern. Ann. Sci. École Norm. Sup. 22, 195-226 (1989) Zbl 0701.14003 MR 1005159

[10] Freixas i Montplet, G.: Généralisations de la théorie de l'intersection arithmétique. Thèse de doctorat, Orsay (2007)

[11] Freixas i Montplet, G.: Heights and metrics with logarithmic singularities. J. Reine Angew. Math. 627, 97-153 (2009) Zbl 1195.14033 MR 2494930

[12] Freixas i Montplet, G.: An arithmetic Riemann-Roch theorem for pointed stable curves. Ann. Sci. École Norm. Sup. (4) 42, 335-369 (2009) Zbl 1183.14038 MR 2518081

[13] Gillet, H., Soulé, C.: Arithmetic intersection theory. Publ. Math. IHES 72, 93-174 (1990) Zbl 0741.14012 MR 1087394

[14] Gillet, H., Soulé, C.: Characteristic classes for algebraic vector bundles with Hermitian metrics I, II. Ann. of Math. 131, 163-203 and 205-238 (1990) Zbl 0715.14018(I) Zbl 0715.14006(II) MR 1038362(I) MR 1043268(II)

[15] Gillet, H., Soulé, C.: An arithmetic Riemann-Roch theorem. Invent. Math. 110, 473-543 (1992) Zbl 0777.14008 MR 1189489

[16] Grothendieck, A.: Éléments de géométrie algébrique: IV. Étude locale des schémas et des morphismes de schémas. Quatrième partie. Publ. Math. IHES 32 (1967), 361 pp. Z Zbl 0153.22301 MR 0238860

[17] Gruber, T., Lekkerkerker, C. G.: Geometry of Numbers. North-Holland (1987). Zbl 0611.10017 MR 0893813

[18] Hartshorne, R.: Algebraic Geometry. Grad. Texts in Math. 52, Springer (1977). Zbl 0367.14001 MR 0463157

[19] Hejhal, D. A.: The Selberg Trace Formula for PSL(2, R), Vols. I, II. Lecture Notes in Math. 548, 1001, Springer $(1976,1983)$ Zbl 0347.10018 MR 0439755

[20] Keel, S.: Intersection theory of moduli space of stable N-pointed curves of genus zero. Trans. Amer. Math. Soc. 330, 545-574 (1992) Zbl 0768.14002 MR 1034665

[21] Keel, S., Tevelev, J.: Equations for $\bar{M}_{0, n}$. Int. J. Math. 20, 1159-1184 (2009) Zbl 1187.14031 MR 2572597 
[22] Knudsen, F. F.: The projectivity of the moduli space of stable curves II, III. Math. Scand. 52, 161-199, 200-212 (1983) Zbl 0544.14020(II) Zbl 0544.14021(III) MR 0702953(II) MR 0702954(III)

[23] Knudsen, F. F., Mumford, D.: The projectivity of the moduli space of stable curves I: preliminaries on "det" and "Div". Math. Scand. 39, 19-55 (1976) Zbl 0343.14008 MR 0437541

[24] Kühn, U.: Generalized arithmetic intersection numbers. J. Reine Angew. Math. 534, 209-236 (2001) Zbl 1084.14028 MR 1831639

[25] Laumon, G., Moret-Bailly, L.: Champs algébriques. Ergeb. Math. Grenzgeb. 39, Springer (2000). Zbl 0945.14005 MR 1771927

[26] Moret-Bailly, L.: La formule de Noether pour les surfaces arithmétiques. Invent. Math. 98, 491-498 (1989) Zbl 0727.14014 MR 1022303

[27] Obitsu, K., Wolpert, S. A.: Grafting hyperbolic metrics and Eisenstein series. Math. Ann. 341, 685-706 (2008) Zbl 1146.30028 MR 2399166

[28] Sarnak, P.: Determinants of Laplacians. Comm. Math. Phys. 110, 113-120 (1987) Zbl 0618.10023 MR 0885573

[29] Schulze, M.: On the resolvent of the Laplacian on functions for degenerating surfaces of finite geometry. J. Funct. Anal. 236, 120-160 (2006) Zbl 1094.58012 MR 2227131

[30] Silverman, J. H.: The theory of height functions. In: Arithmetic Geometry, G. Cornell and J. H. Silverman (eds.), Springer, 151-166 (1986) Zbl 0604.14022 MR 0861975

[31] Soulé, C., Abramovich, D., Burnol, J.-F., Kramer, J.: Lectures on Arakelov Geometry. Cambridge Univ. Press (1992). Zbl 0812.14015 MR 1208731

[32] Szpiro, L. (ed.): Séminaire sur les pinceaux arithmétiques : la conjecture de Mordell. Astérisque 127 (1985) Zbl 0588.14028 MR 08001916

[33] Takhtajan, L., Zograf, P. G.: The Selberg zeta function and a new Kähler metric on the moduli space of punctured Riemann surfaces. J. Geom. Phys. 5, 551-570 (1988) Zbl 0739.30032 MR 1075722

[34] Takhtajan, L. A., Zograf, P. G.: A local index theorem for families of $\bar{\partial}$-operators on punctured Riemann surfaces and a new Kähler metric on their moduli spaces. Comm. Math. Phys. 137, 399-426 (1991) Zbl 0725.58043 MR 1101693

[35] Wolpert, S. A.: Asymptotics of the spectrum and the Selberg zeta function on the space of Riemann surfaces. Comm. Math. Phys. 112, 283-315 (1987) Zbl 0629.58029 MR 0905169

[36] Wolpert, S. A.: The hyperbolic metric and the geometry of the universal curve. J. Differential Geom. 31, 417-472 (1990) Zbl 0698.53002 MR 1037410

[37] Wolpert, S. A.: Cusps and the family hyperbolic metric. Duke Math. J. 138, 423-443 (2007) Zbl 1144.14029 MR 2322683 